Hill, Albert Frederick

The vascular flora of the

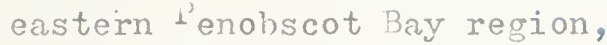
Maine 





\section{VOL. III, $\quad 1919 . \quad$ PART ${ }^{-2 .}$}

\section{P R O C E E D I N G S}

OF THE

\section{PORTLAND SOCIETY}

$\mathrm{OF}$

\section{N A T URAL H I S T O R Y.}

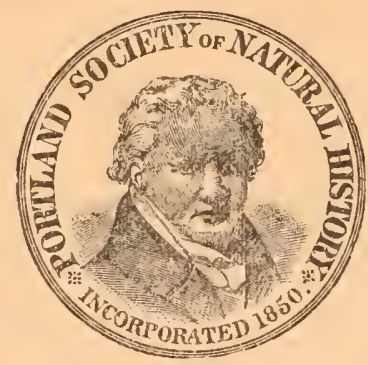

ORGANIZED 1843.

CONTENTS.

II. HILL, ALBERT FREDERICK : Vascular Flora of the Penobscot Buy Region. 



\title{
THE VASCULAR FLORA OF THE EASTERN PENOBSCOT BAY REGION, MAINE
}

\author{
BY ALBERT FREDERICK HILL \\ INSTRUCTOR IN BOTANY AND ASEINTANT GTRATOR OF THE BOTANICAL COLIECTIONE \\ YALE UNIVERAITY \\ GENERAT FEATURES OF THE REGION \\ LOCATION
}

The Penobseot Bay region in Maine is situated on the eoast abont midway between the eastern and western boundaries of the state. Strietly speaking, the temm, "Penobseot Bay", should be used only for that portion of the region lying at the month of the Penobseot River. In general, however, the name is applied to all the territory between the island of Momnt Desert on the east and Roekland on the west. This area lies between meridians $65^{\circ} 25^{\prime}$ and $60^{\circ} 2^{\prime}$ west longitude and parallels $44^{\circ}$ and $44^{\circ} 29^{\prime}$ north latitude. It contains approximately 6.50 square miles, about half of which is water. Of the land, about one-rquarter belongs to the mainland and the remainder to islands. The latter are very numerons and vary in size from the smallest ledges to some whieh eontain as many as twenty-five square miles. (See map.)

It is with the eastern part of this region alone that we shall be concerner. This embraces the townships of Brooklin, on the mainland, and Deer [sle, Stonington, Swans Island, and 
Isle an Hunt, among the islands. With the exception of the latter, which is a part of Knox County, all these towns are in Haneoek County.

\section{Topography}

In its topographical aspeets the eastern Penobseot Bay region presents features more or less common to all that part of the coast of Maine which lies hetween Casco and Machias Bays. The great irregularity of the shore line, the presence of the numerous fjord-like indentations, the deep, narrow channels, and the innumerable islands are all characteristics of a drowned coast. In this type of topography a former land area has undergone submergence, the lower portions of the old river valleys becoming the bays and deep channels, and the hilly uplands giving rise to the many islands. The extreme irregularity of the contours of the present land areas, as well as of those now below the surface of the sea, has been caused by weathering and erosion, clearly in preglacial times. In faet, the entire region represents a peneplain worn down from former highlands by this constant demudation. As a result elevations of any considerable height are extremely rare. Only the few hills with a rockstructure resistant enough to withstand the general wearing down have persisted. Isle au Hant, so named by Champlain, one of the earliest explorers in this region, is one of these residual mountains, or monadnocks. Next to Mount Desert it is the highest island on the Atlantic coast ${ }^{1}$ of the United States. Together with Mount Desert, and the Camden Hills on the mainland to the west, Isle au Haut constitutes the most conspicuons remnant of the old mountains formerly on

1. Shaler, N. S., Geology of the Island of Mt. Desert. 8th Ann. Report Dir. U. S. Geol. Survey, 18S6-7, p. 994. 


the shore. Because of the abruptness with which this island rises from the sea, its actual height of 556 feet is so magnified that the name of "mountain". locally applied to it, is almost justified.

The shores of the outer islands are for the most part rocky and abrupt, in many cases almost perpendicular. Sea cliffs and headlands occur, though never of any great height, wherever the shores are exposed to the constant buffeting of the waves, as on the south side of Swans Island and Isle an Haut. Beaches are common on Deer Isle and the mainland, where the surface is more level. Mud flats and salt marshes are found in tidal estuaries and other protected situations, especially in the shallower indentations. These marshes, however, are small in extent and comparatively scarce, a condition very different from that in more sonthern regions on the Atlantic coast.

The water courses of the region are very insignificant. It most they are small streams a few miles in length, and in the valleys usually obstrueted by glacial drift. Ponds are fairly abundant and belong to three clearly defined types. The majority owe their existence to glacial activity, and these glacial ponds, with but one exception, can be further classed as morainal, a term used by Nichols ${ }^{1}$ to distinguish those lakes "associated with aecumulated glacial debris of any description." In the Penobscot Bay region such morainal ponds occupy the poorly drained depressions or oceur along streams behind obstructions of glacial till. With them are universally associated peat bogs in all stages of development.

Long Pond on Isle an Haut is the only example of a rock basin or scoop lake, one which lies in a depression scooped out of the bed rock itself by the ice. Unlike the other ponds

1. Büll. Torr. Bot. Club, 42: 170 (1915). 
this has rocky shores and no development of swamps or bogs ahout its margins.

The third type of pond is the brackish barrier beach pond, formed by the cutting off of some little indentation of the sea hy the deposition of beach sands and gravels or of waveworn rocks. They ocenr along the more shallow shores on the outer islands where opportmnity is afforded for the neeessary wave action. Is in the case of the morainal ponds these show all stages of transition to swamps and uplands.

\section{Geology}

The rocks ${ }^{1}$ which underlie the eastern Penolseot Bay region are for the most part highly silicions and eonsequently acidic in nature. ${ }^{2}$ Granite is exceedingly abundant, occupying fully three-quarters of the whole area. especially at the higher elevations. Most of the smaller islands are entirely made up of this rock, while on the larger, such as Swans Island and Deer Isle, there are areas many miles in extent. The second most abundant formation, particularly on the mainland and Deer Isle, is a sedimentary schist, "rich in mieaceons minerals and quartz." On some of the outer islands there are small amounts of diorite. diabase, and gabbro. These vary in composition from a "quartz diorite only slightly different from granite to a much more basic rock." On the western side of Deer Isle and on Isle au Hant are found varions volcanics, occurring as flows, breceias, prroclastics, and other surface forms. Sinee these rocks,

1. Statements regarding the rocks of this region are taken from U. S. Geol. Survey, Penobscot Bay Folio 149.

2. If the chemical nature is considered, rocks are grouped as acidic (over $65 \%$ silica), basic (less than $55 \%$ silica), and intermediate (from 55\%-65\% silica). See Merrill, Rocks, Rock Weathering and Soils. New York. 1913; p. 59. 
resulting from the lava flow of old voleanoes, are extremely susceptible to weathering, the ledges and cliffs thereabouts are earred into caves, pinnacles, and many fantistic shapes. The only other rock present in any abundance is serpentine, occurring in the form known as "black marble" in a large tract on the northern end of Deer Isle. This section, it is interesting to note, is the most fertile in the whole area. a condition direetly opposed to that in most serpentine regions. As will be shown later, it is the sole habitat for a few of the common plants of richer soils, plants which for the most part are absent from the rest of the region, since in the sterile acid soils which prevail elsewhere they are unable to find the necessary conditions for their existence.

Unlike conditions in most glaciated districts the rock structure still controls the topography of the Penobscot Bay region, for the surface corering is rery meagre and indeed entirely lacking from the higher places. The soils which do occur are chiefly of glacial origin, although there are a few post-macial deposits. The slacial drift or till, made up of sands and gravels, is thin and irregularly distribnted, ocenrring more abundantly in the valleys. These deposits represent the coarser parts of the sediment which was bronght down by the ice and deposited on the land. The finer portions, on the other hand, were carried on into the ocean and laid down as clay. Most of the present lowlands of the region have a covering of this very fine grained marine elay, but above the 125 foot level and on the steeper slopes this deposit is lacking. Wherever it oecurs the clay is a conspicnous feature of the landseape. for when dry it forms a light gray dust which covers the regetation, and when wet it turns to a very sticky mud.

Directly following the Glacial period, during which the 
foregoing surface deposits were laid down, there took place a period of submergence when the whole coast stood at a much lower elevation than at present. Shaler ${ }^{1}$ gives three features as criteria of snbmergence: (1) the remains of marine animals in stratified drift; (2) the existence of stratified deposits of sands and gravels where their formation conld not be attributed to fresh water lakes; and (3) a topography above high tide level due to marine action. Evidence along these lines is present in the Penobscot Bay region, but since Shaler himself states that the second is the only really acceptable eridence, that alone will be considered. On Isle an Hant heach gravels occur up to a height of 225 feet, ${ }^{2}$ but above 250 feet no wave-washed stones have been found. This would indieate that the land was submerged to a depth of between 240 and 250 feet. In addition the till on the faces and angles of the hills and on the smaller islands has been extensively dennded, a phenomenon which must be referred to marine action. The above facts show that upon the withdrawal of the ice from the immediate ricinity the sea must have stood some 240 feet above its present level, and consequently much of the present land area was under water and the rest extremely isolated. Althongh this condition existed to some extent along the rest of the coast it must have been particularly marked in the Penobseot Bay region, because of the extreme irregularity of the shore line at this point. Both the submergence and the isolation would lead to a limitation of the flora which conld reach these areas while on its northward migration after the Glacial epoch.

From its submerged condition the land has risen by a

1. Recent Changes of Level on the Coast of Maine. Mem. Bost. Soc. Nat. Hist., Vol. II, Part IlI, No. III, 1874.

2. See Stone, G. H., Mon. U. S. Geol. Surv., Vol. 34, 1889, p. 48. 
series of uplifts, some longer than others, to its present elevation-a movement which may still be in progress. The present period of relative quiescence, however, seems to have been mueh longer than any of the preceding halts in the process, and there is considerable speculation as to whether the coast line may not be stationary at the present time or even show sinking again. Owing to the rocky nature of the shores evidence on this point is extremely slight, and the little that does exist is so controlled by local conditions as to be worthless in a eonsideration of the general problem. Indeed, conditions in one locality point toward the subsidence theory, while in others exactly the opposite is the case.

The post-glacial history of the region is as yet very brief. Only a few deposits are present, chiefly beach sands and gravels, swamp muck and peat. As the ocean is constantly eroding the bed-rock and the till and clay, parts of this weathered material are deposited at low elevations in shallow indentations as sand or grarel beaches or as mud flats. On the outer islands where the shores are rocky the beaches are formed, and inland, where the surficial deposits are within reach of the waves, mud flats are the rule. Sandy beaches are rare enomgh to be cmiosities, occurring only along the shores of a very shallow bay on the southern side of Swans Island.

Is has been stated previonsly, the swamp muck and peat have developed in the poorly drained depressions on level surfaces. Here their work of converting the ponds and swamps into dry land is eonstantly going on.

\section{Climate}

It is not to be expected that climatic factors, which are operative only in broad areas, conld render conditions in the 
Penobscot Bay region any different from those in the rest of northern New England; and such is the case. The climate in this district is essentially that of any moist north temperate area, with warm dars and cool nights during the summer. As is nsual in coastal regions, howerer, the proximity of the ocean bring about a much more equable climate than is found inland, and this has an effeet on the vegetation in that the growing season is prolonged. Data from three U. S. Weather Bureau stations on the coast (Eastport, Bar Harbor, Portland) for five years, 1909-1913, show an average growing season of 162 days, while a similar arerage for inland stations (Orono, Millinocket and Greenville) is only 111 dalys. Althongh the precipitation records show very few variations throughout the state as regards the actual rainfall, the coast of Maine, partieularly from Eastport to Penobscot Bar, owing to the prevalence of fogs, is in smmmer an exceptionally moist area. The fogs, caused by the meeting off shore of the warm waters of the Gulf Stream and the iey waters of the Aretic Current, are almost constantly present. Sitnated at the entrance to the Bay of Fundy, the "fog faetory" as it is ealled. Eastport shows a response to the excessive moisture in that it has a lower temperature in summer than any other station in the state for which records are available. The same condition oecurs in the Penoloseot Bay region, but no official records are available. The constant dampness of the atmosphere, together with the effect of the cold water which washes the shores, creates eonditions along the onter islands and coast verer similar to those in more aretic regions, and thms affords a habitat for aretic plants. This boreal region extends from Eastport to Penobseot Bay and even beyond, but it is much less marked west of our area. 


\section{Previous Botaxicat Work}

Although the island of Mount Desert, on the eastern border of the Bay, and several regions to the westward have been earefully studied hotanically, the Penobseot Bay seetion of the Maine Coast has been much negleeted.

Previons to 1908 only desultory collections were made. Francis L. Harvey, Ora Wr. Kinight and others of the Maine botanists made occasional trips among the islands, and a few of their plants, together with seattering specimens obtained by summer visitors, have found their way into varions herbaria.

In 1908 Professor Arthm H. Graves made a study of the woody plants at Brooklin ${ }^{1}$ and collected specimens, now deposited in the herbarimu of Yale University. Nore reeently Mr. Arthur H. Norton has collected extensively along the Maine coast ${ }^{2}$ and a few specimens from eastern Penolscot Bay are to be found among his plants in the herharimm of the Portland Soeiety of Natural History.

Several private collections have been made in the region, notably that of Mrr. Nathamiel T. Kidder in the vicinity of Isle au Haut. P'rofessor J. C. Arthur of Purdue Unirersity spent several sealsons on the latter island and, although he colleeted but few specimens, he reeorded 315 species as occurring in that locality. No systematie study of the flora, however, has been made until the present investigation.

\section{Acknowlengalexts}

I wish at this time to express my indebtedness to Professor Merritt L. Fernald, who has kindly verified the majority of

1. Graves, A. H. Woody Plants of Brooklin, Maine. Rhodora 12: 173-184 (1910).

2. Norton, A. H. Some Noteworthy Plants from the Islands and Coast of Maine. Rhodora 15:137-143 (1913). 
determinations of specimens; to Professor Karl M. Wiegand, Doctor Ezra Brainerd, and Mr. F. Tracy Hubbard, who have kindly identified certain perplexing genera: to the curators of the Cray Herbarimm and the herbarium of the Portland Society of Natural History, who have extended every courtesy to me in placing the collections under their charge at my disposal; and to Professor J. C. Arthur, Mr. Nathaniel T. Kidrler, Mr. Charles L. Curtis and all others who at various times have given invaluable assistance in the securing of data or the collection of the specimens themselves.

\section{THE VASCLLAR PLANTS OF THE REGTON}

In the following list of the vascular plants of the eastern Penobscot Bay region in Maine, the nomenclature of the Pteridophyta follows Christensen's Index Filicum'; while that of the Spermatophyta follows the Seventh Edition of Grav's Мanual, save where new species or varieties and nomenclatorial changes have been made since its publication. In the latter instances, wherever possible, the manual name is wiven in brackets.

The records are based for the most part on specimens collected by the writer, a complete set of which has been deposited in the herbarium of the New England Botanical Club. Further records made by other collectors, are included whenever they are corroborated by herbarium specimens. Among these may be mentioned the collections of Graves, Kidder, and Torton ${ }^{4}$, referred to previously. In

1. Christensen, C. Index Filicum. Copenhagen. 1906.

2. Robinson, B. L., and Fernald, M. L., Gray's New Manual of Botany, Ed. 7. New York. 1908.

3. Deposited in the herbarium of Yale University.

4. Deposited in the herbarium of the Portland Society of Natural History. 
addition a few plants are listed of which no specimen has been seen, but which appear in well-authenticated lists, such as Arthur's unpublished list of Isle an Haut plants. These latter records are denoted by an asterisk (*).

DIVISION I

PTERIDOPHYTA

\section{POLTPODIACEAE}

Oxoclea sexsibilis L. Tery common in wet places. Tariable in form, often approaching var. obtusilobata (Sehkuhr.) Torr.

Driopteris thenypteris (L.) Gray. [Aspidium Thelypteris (L.) Sw.] Common in swampy meadows and logs. Dryopteris noveboracexse (L.) Gray. [Aspidium noveboracense (L.) Sw.] Common in moist open woorls and along roadsides.

Dryopteis araginalis (L.) Gray. [Aspidium marginale (I.) Sw.] Rich woods near Lily Pond, Sonth Deer Isle (No. 3070).

Driopteris cristata (L.) Gray. [Aspidium cristatum (L.) Sw.] Abundant in wet roods.

Driopteris spixulosi (Mïll.) Kimtze. [Aspidium spinulosum. (O. F. M[ïller' Sw.] Very common in wet woods and thickets.

var. internedia (M[uhl.) Underw. [Aspidium spinulosum, var. intermedium (Muhl.) Eaton.] Abundant in similar situations.

var. anericaxi Femald. ${ }^{1}$ [1. spinulosum var. dilatatum of Am. auth., not Hook.] Mroist woods, Brooklin (No. 1791): Isle an Haut (No. 1708).

1. Rhodora 17: 48 (1915). 
Dryopteris Phegopteris (L.) C. Chr. [Phegopteris polypodioides Fée.] Common in cool rich woods.

Drioptenis Liscataxa C. Chr. [Phegopteris Dryopteris (L.) Fée.] Abundant in damp woods.

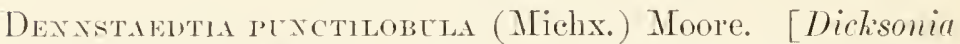
punctilobuln (Michx.) Gray.] Abundant in open woods and fields, often growing in dense colonies several rods in diameter.

Athymua Filix-fenixa (L.) Roth. [Asplenium Filixfeminu (L.) Beruh.] Common in moist woods and thickets.

Asplandum Trichomaxes L. Crevices of serpentine cliff, Little Deer Isle, Deer Isle (No. 2580).

Adiaxtua pedatual L. Rich moist woods on serpentine, Reach, Deer Isle (Nos, 1945, 2613). Although common in the western and northern parts of the state and in adjacent Canada, the maiden hair fern has not been reported previously from the coast, east of the Kennebec River.

Ptemindar aquilixiar (L.) Kuhn. [Pteris aquilina L.] Terv common in dry open woods and clearings.

Wondsia lluexsis (L.) R. Br. Crevices of serpentine ledges and cliffs, Little Deer Isle. Deer Isle (No. 2596).

Polyponita velgare L. Occasional on ledges or in rocky woods.

\section{OSMIUNDACEAE}

Osmexpa regalis L. Occasional in wet woods, swamp unargins or thickets.

Osandida Chatoniaxa L. Common everywhere in low gromind. 
Osurpar cincamonea T. Abundant in swampy meadows and wet woods.

\section{OPHIOGLOSSACEAE}

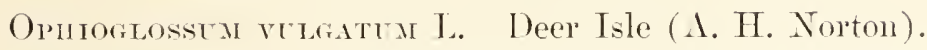

Botrichiras simplex E. Hitcheock. Rich wonds, Gray's Cove, Reach, Deer Isle (No. 2765).

Botrychina raxostan (Roth) Aschers. Sumy hillside on serpentine, Little Deer Isle. Deer Tsle (C. L. Curtis).

Botrychica obliquar Mubl. Tnfrequent. Dry open wonds, fields and pastures.

\section{EQUISETACE.IE}

Equtsetua arvexse L. Common in moist, sandy or gravelly places.

Equisetula syuvatiocal L. Frequent in moist wonds and shaded spots.

Equisetua futratile L. Rare. Shallow water. muddy shore of Torrey Pond, Deer Isle (Nos. 1957, 2.564).

\section{LYCOPODIACE.IE}

Lrcopontul Selatio L. Rich cool woods on serpentine, Reach, Deer Isle (No. 1592). An umusual station for this aretic-alpine species of cold hogs and exposed situations.

Lycopodura Lucintera Michx. Occasional in low wet mossy woods.

Lrcopodiar txexpatrar T. Abundant on sphagnum mat, Stockbridge Pond, Swans Island (No. 2323): Marshall's Island ( 1. H. Norton). 
Lycoponfun axpotixea L. Common in dry woods.

var. Acrifolicar Fernald ${ }^{1}$. Dry open woods, Naskeag Point. Brooklin (Nos. 1429, 2999); damp woods on .Jerusalem Mt., Isle au Haut (No. 118.3).

Lycopontur clabatra L. Very common in dry open moods. var. megastachyox Feruald \& Bissell ${ }^{2}$ [var. monostachyon Grer. \& Hook]. Similar situations, Naskeag Point, Brooklin (No. 3003); Seal Cove, Swans Island (No. 2661) ; Dumham's Point, Deer Isle (Nos. $2215,2815)$.

Lrcopontum obsctray T. Abundant in rich woods. This species, the most frequent of the club mosses, is extremely variable, showing all gradations to var. dendroideum (Michx.) T. C. Eaton.

var. dextrometir (Michx.) D. C. Eaton. Occasional in rich woods.

Lycoponem complaxatede L., var. flabelliforme Fernald. Occasional in open woods. Naskeag Point, Brooklin (No. 235) ; Tittle Deer Isle, Deer Isle (No. 2592); Greenlaw District, Deer Isle (No. 2835).

Ircopodiex tristachytar Pursh. Dry woods near Lily Pond, South Deer Isle (No. 3069) : Central Tract, Brooklin (A. II. Graves): Naskeag Point, Brooklin (Miss Tyler ).

\section{SELAGTNELTACEAE}

Selaginelat rupestris (L.) Spring. Dry serpentine cliffs and outerops, Little Deer Isle, Deer Isle (No. 2594).

1. Rhodora 17: 124 (1915).

2. Rhodora 12: 53 (1910). 


\section{TAXACEAE}

Thxus caxadexsis Marsh. Oceasional in deep wet woods.

\section{PINACEAE}

Prnus Strobus L. Frequent in dry woods. Formerly this tree was much more ahundant but it has been almost exterminated by lumbermen. A few large speeimens are to he found in the deeper woods, but usually the trees are young and only a few feet (10-20) in height.

Pines rigidat Mill. Oecasional on barren soil. The piteh pine, which is at almost its northem limit, is usually found only in small isolated areas. However, on the sterile slopes and summit of MIt. Champlain, Isle an Haut, it is verv abundant.

Pixes resixosa Ait. Infrequent. Near Sedgwick road, Brooklin (A. II. Graves) : dry barren soil, North Deer Isle (No. 2789); *Isle au Hant ( T. T. Kidder).

Larix larictica (DuRoi) Kól. Abundant in wet woods and sphagnum bogs.

Picen Chandexsis (Mill.) BSP. Common on uplands.

Picea rubra (DuRoi) Dietr. Very abundant on uplands. This species together with the preceding and Abies balsamea make up the bulk of the forests of this region. Stunted specimens are common on the outer headlands and on Mrt. Champlain, Isle an Hant.

Picea mariaxa (Mill.) BSP. Frequent in sphagnum bogs. Abies balsairea (L.) Mill. Abundant on uplands.

Tsuga caxadexsis (L.) Carr. Very rare. Low woods, Gray's Cove, Deer Isle (No. 2190); Flyes Point, Brooklin (A. H. Graves). 
Thirda occhextalis L. Frequent in wet swampy woods.

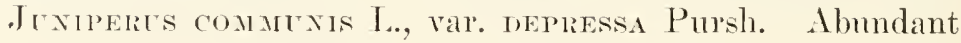
in dry sterile fields.

Juxiperis uorizoxtalis Moench. Common on rocky headlands on the onter islands but rare inland. Inry fields, Naskeag Point, Brooklin (Miss Gilbert); Flyes Point, Brooklin ( 1. II. Graves).

\section{TIPHACEAE}

Trpisa Latifolia T. Frequent in swamps and pond margins.

\section{SPARGANIACEAE}

Spargaxicar americaxtar Nutt. Occasional on muddy pond shores and along streans. Deer Isle and Isle an Taut.

var. axproctabea (Engelm.) Fermald ef Eames. Oeeurring with the typical form.

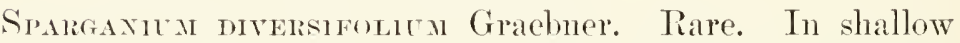
water, muddy shores of Lily Pond, South Deer Isle, Deer Isle (No. 1848); ditches in swampy meadow, North Brooklin (No. 2909).

rar. Ac.rtue (Becby) Fernald \& Eames. MIuddy bog hole in pasture. Brooklin (No. 1030) ; North Brooklin (No. 2909a).

*Sparandear manum Fries. Tnfrequent. Isle an Hant (J. C. Arthme).

\section{NA.JADACEAE}

Potanocietox rataxs-L. Floating in deep water. Torrey Pond, Deer Isle (No. 25,60a).

Pothagegton Onkeshanes Roblins. Common in ponds in sphagnum hogs. 
Potamogeton bupleuroides Fernald. Shallow water at edge of brackish pond, Otter Ponds, Swans Island (No. 2341).

Potmiogetox manompics Raf. Rare. Torrey Pond, Deer Isle (No. 1989).

Ruppia maritima L., var. Loxgipes Hagström. ${ }^{3}$ Brackish ponds, Swans Island.

Zostera mariva L. Abundant in shallow water on muddy shores, usually below low tide level.

\section{JUNCAGINACEAE}

Triglocim maritima L. Common on muddy shores and in salt marshes.

\section{ALISMACEAE}

Sagittaria latifolia Willd. Rare. Shallow water, shores of Torrey Pond, Deer Isle (No. 1964). Tariable, showing transitions to forma oвтusa (Mruhl.) Robinson (No. 1968), and forma Gricilis (Pursh) Robinson (No. 1966). *Sagittaria gramixea Miehx. Isle au Hant (N. T. Kidder).

\section{GRAMINEAE}

Panicua capillare L. Rare. Clearing in dry woods, Brooklin (No. 1757).

Panicum pinfanelpincuar Bernh. Dry woods near Sunset, Deer Isle (No. 1865).

1. Botaniska Notiser (1911) 138. See Fernald, M. L., and Wiegand, K. M., Rhodora 16: 119-127 (1914). 
Paxicear Trerneri Seribn. Common in open woods, elearings and along roadsides near serpentine quarry, North Deer Isle, I leer Isle.

Paxicta borfale Nash. Occasional in damp fields.

Paxicur utachecaf Ase. Dry elearings in rich woods, Torth Deer Tsle (Nos. 2002, 2600).

var. Fascictlatian (Torr.) Hubbard. ${ }^{1}$ [rar. silvicola Hitche. \& Chase.] Common everywhere in dry woods and clearings.

Paxice mimpicatum Seribn. Abundant in meadows and dry fields.

Paxictar srevillostar Ashe. Rare. Clearings and open woods, Brooklin.

Echixocirod crescidili (L.) Beaur. Frequent in waste places and eultivated ground.

Sethlia ridalca (L.) Beaur. Rare. A weed in cultivated ground at Taskeag Point, Brooklin (No. 1372).

Setaria virinis (I.) Beaur. Oceasional in cultivated ground and waste places. Shore of Mill Pond, Deer Isle (No. 2212).

Setaria italica (L.) Beaur. subsp. stramineofictota Hubbard, var. Hosti Hubb., subvar. Metzgeri (Körn.) Hubb. ${ }^{2}$ Eseaped from cultivation. Locally abundant at several places on Naskeag Point, Brooklin.

Phalaris artixifacea L., var. picta L. Commonly enltivated and often an escape. Roadside thickets on Sedgwick-North Brooklin road, Brooklin.

1. Rhodora 14: 171 (1912).

2. American Journal of Botany 2: 191 (1915). 
Axthoxixthum onoritur L. Tery common in meadows and fields. A peculiar antumnal form with long densely pilose leaves was collected in a dry clearing on Isle au Haut (No. 2433).

Hierocilod odorata (T.) Wahlenb., var. Fragraxs (Willd.) Richter. ${ }^{1}$ Frequent in salt marshes and brackish meadows.

Oryzopsis asperifolia Michx. Occasional in dry woods and clearings, Deer Isle.

Brachyemtrum erectum (Schreb.) Beaur. Frequent in low woods. Swans Island and Deer Isle.

Pillecar pratense L. Abundant in fielels, roadsides and cultivated ground.

Sporoboles rriflores (Mruhl.) Scribn. \& Merr. Mroist. open woods. North Brooklin (No. 2902); *Tsle au Haut (J. C. Arthur).

Agrostis alba L. Abundant everrwhere in meadows and fields.

var. rulgaris (With.) Thurb. Common in all open places. This variety and the typical form are the most abundant of the wild meadow grasses used for hay.

var. ARIstata Gray. Rare. Shaded bank of brook near Herricks Bay, Brooklin (No. 1109).

var. maritian (Lam.) G. F. W. Mẹ. Frequent in salt marshes and brackish meadows.

Agrostis myenalis (Talt.) BSP. Common in clearings and dry fields.

Agrostis perencaxs (Walt.) Tuckerm. Occasional in moist woods or meadows.

1. Plantae Europeae 1:31 (1890). See Fernald, M. L., Rhodora 19: 152 (1917). 
Agrostis canixa L. Rare. Wet meadow at Swans Island Head, Swans Island (No. 756), apparently perfectly naturalized.

Camamagrostis caranexsis (Michx.) Beamy. Common in wet places, especially near the shore.

Ammophina arexaria (L.) Link. Occasional. Sandy beaches on sonth side of Swans Island; shores of Flyes Island and Naskeag Point,Brooklin.

Cinsa latifolia (Trev.) Griseb. Frequent in moist open woods.

Trisetrar spicatum (T.) Richter, var. molle (Michx.) Piper." Common on rocky headlands, Greenlaw's Neck, Deer Isle (No. 2807) ; dry open woods, Naskeag Point, Brooklin (No. 2980).

Descimampsia flexuosa (T.) Trin. Common on headlands, exposed summits and in dry clearings. A very delicate form is oceasionally found in moist woods.

Arexa sativa L. Frequent along roadsides and in waste places and cultivated gromnd.

Daxthonia spicata (L.) Beanr. Very abundant in all open sterile localities. This species and Festuca rubra are the common grasses on headlands, exposed onterops and other places where the soil covering is very thin.

Daxthonia compressa Aust. Occasional on headlands or in sterile soil elsewhere.

Spartixa Michariana Hitche. Frequent at the edge of beaches or in salt marshes.

Spartina alterifflora Loisel. " [Spartina glabra Minhl.,

1. Contrib. U. S. Nat. Herb. 11: 125 (1906). See Fernald, M. L., Rhodora 18: 195-198 (1916).

2. Fl. Gall. 2: 719 (1807). See Fernald, M. L., Rhodora 18: 178 (1916). 
var. alterniflora (Loisel.) Merr.] Common on muddy beaches and along the edge of salt marshes.

Spartina patexs (Ait.) Muhl. Abundant in salt marshes and occasionally on beaches.

Dictylis glomerata L. Occasional in fields. Naskeag Point, Brooklin.

PoA axrea L. Occasional in ruderal ground.

Pon compressa T. Common in dry sterile soil, usually in the open. Occasionally found on sandy beaches.

Poa grauca Tahl. Rare. Damp rich woods near serpentine quarry, North Deer Isle (No. 2609). This plant needs further study but seems to approach most elosely this species.

Poa triflora Gilib. Frequent in wet meadows and along streams.

Pon pratensis L. Common in fields and pastures.

Glyceria obtusa (Muhl.) Trin. Rare. Swampy margin of Lily Pond, South Deer Isle (No. 1850).

Guyceria caxanexsis (Michx.) Trin. Very abundant in swamps and wet meadows.

Glycerta laxa Scribn. Frequent in swamps.

Glycerta nemvata (Willd.) Trin. Common in moist woods and fields.

Puccineluta patpercula (Holm) Fernald \& Weatherby var. Alaskana (Seribn. \& Merr.) Fernald \& Weatherby. ${ }^{1}$ Abundant on beaches and in brackish meadows. Tery variable in size.

1. Rhodora 18: 18 (1916). 
Festcai rubra L. Very abundant in dry sterile soil.

Festrca elatior L. Wet swampy woods, Naskeag Point, Brooklin; swampy roadsides, Reach, Deer Isle (No. 2155): "Isle au Haut (J. C. Arthur).

Bromes ciliates L. Occasional in wet thickets or roadside ditches.

Arropyrox rerexs (L.) Beaur. Very common on beaches and occasional in fields or along roadsides. In the former habitat at least this species appears indigenous. Occurring in many forms.

var. PILosum Scribn. ${ }^{T}$ Occasional on muddy shores on the outer islands.

Honderar jubatum L. Frequent at the edge of beaches. Deer Isle and Isle an Hant.

Horderat velgare I. Occasional. Roadsides and waste places.

Eryates virginicts L. Common along shores.

var. hinsteiglenis (Serijon.) Hitche. Oceasional in elearings and open woods.

Elyales aliexarues L., var. villoses E. Mey." Abundant on beaches and rocky shores. In this region this speeies replaces Ammophila arenaria as the most common grass on beaches.

\section{CYPERACEAE}

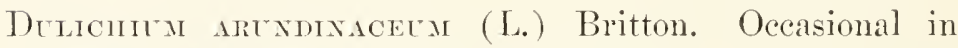
swampy meadows or on pond shores.

Eleocharis obtusa (Willd.) Sehultes. Common in muddy ditches and bog-holes in meadows.

1. Fl. Mt. Des. Isl., 183 (1894).

2. Pl. Labrador 20 (1830). See St. John, H., Rhodora 17: 9S.103 (1915). 
Eleocitaris palestris (L.) R. \& S. Oecasional on swampy shores, either fresh or brackish.

var. crafresscexs (I'illd.) Gray. Boggy meadow near Swans Island Head, Swans Island ( No. 735).

Erfocilaris aciculatis (L.) R. \& S. Rate. Mruddy shore of Lily Pond, Deer Isle (No. 25\%9).

Ereocharis texuts (Willd.) Schultes. Frequent in wet ground.

Scmpes americaxis Per.. Tnfrequent. Brackish shores of the Otter Ponds, Swans Island (No. 155t): Isle au Hant (Miss Furbish, in Herb. X. E. Bot. Club).

Scripls valints Tahl. Occasional in shallow water of ponds. Torrey Pond, Deer Tsle (No. 1979) : Long Pond, Isle au Hant (J. C. Arthur); barrier beach pond. Red Point, Swans Tsland (No. 287s).

Scirpls campestris Britton, var. patedosis (A. Nelson) Fernald. Abundant on brackish muddy shores and on salt marshes.

var. xovie-txglate (Britton) Fernald. Oceasional in brackish pools. Fles Point, Brooklin (No. 1348); Gray's Cove, Deer Isle (No. 1942).

Scripts mitrotinctes Fernald. Rare. Swampy meadow near Duek Harbor. Jsle an Hant (No. 1639).

*Scurpus Atrorirexs Mulhl. Tsle au Hant (J. C. Arthur).

Sompts abonglaxts Harper. Frequent in wet ground.

Scirpers cyperixes (L.) Kunth. Rare. Marshy ground near Rich's Cove, Isle an Haut.

var. Pelits Fernald. Common in wet meadows and swamps.

rar. coxdexsites Fernald. Swampy margin of Long Pond, Isle au Haut (No. 3055). 
Scirpus pedicellatus Fernald. Infrequent. Isle au Hant; Swans Island (Miss Furbish, in Herb. N. E. Bot. (lub).

Scirpes atrocixctes Fernald. Abundant in bogs and swampy places.

Eriophorear callitrix Cham. Common in sphagnum bogs. Deer Isle and Swans Island.

Eriopholida texelutar Nutt. Frequent in bogs and wet meadows.

Eriophorca virini-carixatum (Engelm.) Fernald. Rare. Boggy meadow on Kimballs Island, Isle au Haut (No. $1619)$.

Emophomear viremicua $\mathrm{I}$. Abundant in sphagnum bogs and swamps.

Rrxchospona fusca (L.) Ait. f. Rare. Sphagnum bog at Stockbridge Pond, Swans Island (No. 1537).

Rixchospora alba (L.) Tahl. Tery eommon in sphagum bogs.

Cramuar matiscomes (Mulll.) Torr. Rare. Shallow water at outlet of Torrey Pond, Deer Isle (No. 197S).

Carex scoparia Schkuhr. Abundant everywhere in dry soil.

var. moniliforms Tuckerm. Oeeasional in similar loealities.

var. condexsa Fernald. Common in dry soil.

Carex Craweondi Feruald. Frequent in dry fields.

var. vigexs Fermald. Dry pasture, Flyes Island, Brooklin (No. 298s).

Carex stramnea Willd. Dry rieh woods, Gray's Cove, Reaeh, Deer Isle (No. 275s). 
Carex homatiodes Fernald. Frequent on brackish shores. Swans Island and Brooklin.

var. invisa (W. Boott) Fernald. Rare Brackish soil, Warren Point, South Deer Isle (No. S64).

Carex foexea Willd. Dry fields and meadows, Torrey Island, Eggemoggin Reach, Brooklin (No. 284s).

*Carex adesta Boott. Isle au Hant (J. C. Arthur.).

Carex exilis Dewey. Infrequent in sphagnum bogs. Swans Island and Isle an Haut.

Carex ecirivata Mnur." [Carex stelluluta Good.] Ocersional in low ground.

var. ormantha Femald. ${ }^{2}$ [C. stellulata Good., var. ormantha Fernald.] Rare. Swampy meadow, Dunham's Point, Deer Isle (No. 2283).

var. Excelsior (Bailer) Fernald. ${ }^{2}$ [C. stellulata Good., var. excelsior (Bailey) Fernald.] Rare. Sphagnum bog, Naskeag Point, Brooklin (No. 1458).

var. Cephataxtha Bailey." [C. stellulata Good., var. cephatantha (Bailey) Fernald.] Abundant in bogs and meadows.

var. Axgustata (Carey) Bailey. $\quad$ [C. stellulata Good., var. angustata Carer.] Common in moist woods and low ground.

Carex sterilis Willd. Common in sphagnum bogs.

Carex caxescens L. Rare. Wet meadow near Swans Island Head, Swans Island (No. 755); sphagnum bog, Central Tract, Brooklin (No. 104S).

1. Prodr. Fl. Goth. 76 (1770). See Fernald, M. L., Rhodora 19:154 (1917).

2. Proc. Am. Acad. 37: 483, 484 (1902).

3. Mem. Torr. Bot. Club 1: 58, 59 (1889). 
var. strbolideen Laestad. Swampy woods, Fast Point, Swans Island (No. 2857).

var. mis.uncta Fernald. Very common in wet meadows and bogs.

Carex brexpescexs Poir. Abundant in dry roods and fields.

Chrex trisperma Dewey. Frequent in sphagnum bogs.

var. Billixasir Knight. Oceasional. Bog at Torrey Pond, Deer Isle (Nos. 2179, 2844); near Duck Harbor, Isle an Hant (No. 1635).

Carex texella Schkulhr. Rare. Cool wet roods, Naskeag Point, Brooklin (No. 1436).

Caliex stipata Mulhl. Frequent in wet places near shore.

Carex maritima O. F. Mneller. Common in salt marshes and on beaches.

Calisx salixa Wahlenb, var. kattegatexsis (Fries) Ilmq. ${ }^{1} \quad$ [C. salina, rar. cuspidata Wahlenb. $]$ Rare. Brackish marsh, Naskeag Point, Brooklin (No. 634).

Carex crinita Lam. Infrequent in wet places.

var. graxtha (Schwein.) Schwein. \& Torr. Common along streams and in wet meadows.

Carex Goonexowi .T. Gay. Very common in wet places. An exceedingly variable species.

Carex stricta Lam. U'neommon. Sphagnum bog, Brooklin (No. 1767 ); *Iste au Haut (.J. C. Arthur).

Carex parciflora Lightf. Frequent in sphagnum bogs. Swans Island and Isle an Hant.

1. Hartman, C. J. Handbok i Skandinaviens Flora. ed. 11:466 (1S79). See Rhodora 14: 108 (1912). 
Carex Leptalea Wahlenb. Oecasional in moist soil. Reach, Deer Isle (No. 2164); wet meadow, East Point, Swans Island (No. 2879); *Isle an Hant (J. C. Arthur).

Carex gracildiara Schwein. Locally common in moist woods, Deer Isle.

*Carex deflexa Hornem. Isle an Haut (J. C. Arthur).

Carex comanns Bailey. Rare. Dry rich woods, Gray's Cove, Reach, Deer Isle (No. 2759).

Carex varia Muhl. Infrequent in dry open woods. Brooklin and Deer Isle. "Woodland paths, Isle an Hant (J. C. Arthmr).

Carex novae-axglate Schwein. Frequent in dry woods.

Carex pexpsplaxica Lam. Rare. Woods near Lily Pond, Deer Isle (No. 257\%).

* Carex panicea L. Isle an Hant (J. ( . Arthur).

Caliex pallescexs L. Common in fields and meadows.

Carex palpercula Michx. var. mriata (Tahlenb.) Fernald. Very eommon in sphagnum bogs.

Carex laxiflora Lam. Rare. Rich woods, Gray's Cove, Reach, Deer Isle (No. 2772).

Carex coxoldea Schkuhr. Occasional in dry fields.

Carex flara L. Loeally common in wet woods and meadows. Torrey Pond, Deer Isle (Nos. 217t, 2193).

Carex Oederi Retz., var. pumila (Cosson d Germain) Fernald. Common on headlands and oeeasional in moist places inland.

Carex arctata Boott. Occasional. Dry open woods, West Stonington, Deer Isle (No. 1852); woods near Torrey Pond, Deer Isle (No. 285.i).

Carex nebilis Michx., var. Rudeet Bailey. Frequent in dry woods and clearings. 
Carex scabrata Schwein. Uncommon. Wet mossy woods, Naskeag Point, Brooklin (Nos. 271, 978) ; in leaf mould of moist woods, North Brooklin (No. 2926).

Carex filfformis L. Oceasional. Sphagnum bog, outlet of Goose Pond, Swans Island (No. 1492); muddy shores of Torrey Pond, Deer Tsle (Nos. 19S3, 2165).

Carex Pserdo-Cyperis I. Rare. Wet swampy meadow, Tumham's Point, Deer Tsle (No. 216 T).

Carex rurida Wahlenb. Abundant in swamps and wet places generally.

Carex intumescens Rudge, var. Fernaldi Bailey. Common in wet mossy woods.

Carex folliculata T. Oceasional in sphagnum bogs and meadows.

Carex resicarla L., var. oferca Fernald. Rare. Swampy meadow, Gray's Core. Deer Isle (No. 2570).

Carex rostrita Stokes. Frequent in sphagmum bogs. Common in shallow water. Torrer Pond, Deer Isle (Nos. $1967,2563)$.

var. ctuiculata (Boott) Bailep. Rare. Sedgy swamp, Flyes Island, Brooklin (No. 2986) ; sphagnum bog, Minturn. Swans Tsland (No. 1493); "Tsle au Haut (J. C. Arthur).

\section{ARACEAE}

Arisama triphylutu (L.) Schott. Infrequent in wet swampy woods.

Caliad palustris L. Oecasional on boggy shores. Deer Isle; "Infrequent, Isle au Haut (J. C. Arthur).

Symplocarpis foetidus (L.) Nutt. Frequent in swampy woods. 


\section{ERIOCAULACEAE}

Eriocallon septaxgulare With. ${ }^{1} \quad$ [Eriocaulon articulatum (Huds.) Morong.] Frequent on muddy pond shores, often in deep water. Swans Island, Deer Isle, Isle an Hant.

\section{XTRIDACEAE}

Xrpis moxtana Ries. Locally abundant in sphagnum bog at Stockbridge Pond, Swans Island (No. 2368).

\section{PONTEDERIACEAE}

Pontenena cordata L. Rare. In shallow water, muddy shores of Lily Pond, South Deer Isle (Nos. 1S14, 2S41).

\section{JTNC.ICEAE}

Junces bufonits I. Common on beaches and dry soil inland.

Juxcus Geraror Toisel. Abundant on muddy shores and in salt marshes.

Juxcus texus Willd. Frequent in dry fields and pastures.

Juncus Greenet Oakes \& Tuckerm. Rare. Dry sterile soil, Naskeag Point, Brooklin (Nos. 212, 2970). *Infrequent, Isle au Haut (J. C. Arthur).

Juxces Balticus Willd, var. Littoralis Engehm. Frequent in brackish marshes and in damp mearlows near the shore.

1. Withering's Bot. Arr. Veg. 2: 784 (1776). See Robinson, B. L., and Fernald, M. L., Rhodora 11: 40-41 (1909). 
Juxcts effotsts L., var. compactes Lejeme et Courtois. Infrequent. Swampy meadows, Deer Isle, Isle au Haut. rar. soldters Fernald d Wiegand. Infrequent. Strans Island (Miss Furbish in Herb. N. E. Bot. Club); Wet meadow, Gray's Core, Deer Isle (No. 1917).

rar. Prtaer (Laharpe) Feruald \& Wiegand." Common on wet ground.

Juxces biertcacdates (Engelm.) Fermald. Abundant in muddy places. A very variable species in this region.

Juncers caxadexsis .J. Gay. Oceasional in boggy meadows. Juxces pelocarpus Mey. Infrequent. Brackish marshes, Brooklin and Isle an Haut.

Juxces militaris Bigel. Uncommon. Shallow water, Goose Pond, Swans Island (No. 2678); *Long Pond, Isle an Hant (J. C. Arthur).

Junces articulates L. Occasional. Muddy roadside ditches, North Brooklin (No. 2914); "Isle au Hant (J. C. Arthur).

Luzila saltuexsis Fernald. Occasional in dry open woods, Deer Isle (Nos. 2176, 25t8).

Luzula Caxipestris (L.) DC., var. meltiflora (Ehrh.) celak. Abundant in fields and meadows.

\section{IILIACEAE}

Oanesia sessilifolia (L.) Wats. Infrequent. Dry open woods, Deer Isle.

1. Rhodora 12: 90 (1910).

2. 1. c. 92 . 
Hemenocallis felwa L. Roadsides and fields. Escaped from cultivation.

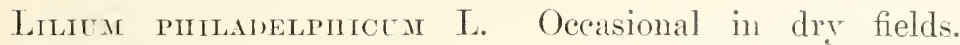
Brooklin and Deer Isle.

Limidar cuxanexse L. Infrequent. Teadows near shore. Brooklin and Deer Isle. I form with orange colored flowers was collected in a moist alder thicket at Gray's Core, Deer Tsle (No. 2s61a).

Clintonla borealis (Ait.) Raf. Very common in rich woods.

Simlacina rickiosa (L.) Desf. Rare. Thicket at edge of woods, Naskeag Point, Brooklin (No. 1456).

Sailacixa stellata (L.) Desf. Pickerings Island, Deer Isle (F. L. Harvey in Herb. Gray).

Samlacisa trifolia (L.) Desf. Abundant in sphagmm bogs.

Manaxthencu candenses Desf. Abundant in moist open woods.

Streptores anplexifolics (L.) DC. Rare. Banks of ravine in damp woods near Eggemoggin Reach, Brooklin (No. 995).

Streptopes rosecs Michx. Frequent in cool rich woods.

Polygonatear biflorial (Walt.) Ell. Rich woods near lake, Isle an Haut ( $\mathrm{N}$. T. Kidder).

Medeola virginiana L. Frequent in dry open woods.

Trilcium uxdelatur L. Occasional in damp woods.

\section{IRIDACEAE}

Iris versicolor I. Common in wet places along shores and in meadows inland. 
Iris setosa Pall., var. canadensis Foster. Rare. Crevices of rocks on headlands, Black Point, Swans Island (Nos. $2337,2705)$. This station is a new southward extension for this arctic species. The previous southern limit has been Little I)uck Island, off Mount Desert. ${ }^{1}$

Sistincintid axgerstifolitar Mill. Very eommon in fields and pastures.

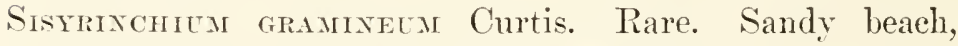
Tonthaker's Cove, Swans Island (No. 2362).

\section{ORCHIDACEAE}

C'rpmentum acacle Ait. Infrequent. Dry rich woods.

Mamexaria milatata (Pursh) Gray. Rare. Rich woods near serpentine quarry, North Deer Isle (No. 2144).

Habexaria clavellata (Miehx.) Spreng. Common in moist open ground.

Habexaria obtisata (Pursh) Richards. Cliffs near Morris' Mistake, Isle an Hant ( $\mathrm{N}$. T. Kidder).

Habevalia Lacera (Miehix.) R. Br. Occasional in moist fields.

Habexaria Istcones (I.) Sw. Infrequent. Swampy meadows. Brooklin and Swans Island.

Habexaria fimbriata (Ait.) R. Br. Common in wet thickets and fields. I form with pure white flowers was collected in damp woods, Gray's Cove, Deer Isle (No. 286.3).

Pogonia ophoglossomes (L.) Ker. Abundant in sphagnum bogs.

Calopogon prleheldts (Sw.) R. Br. Tery common in sphagnum hogs.

1. See Norton, A. H., Rhodora 15: 138 (1913). 
Aretingsa bulbosa L. Infrequent in bogs, Swans Island. *Isle an Haut (N. T. Kidder). A form with pure white flowers was collected in the bog at Stockbridge Pond (No. $1583)$.

Spiraxtires cirarilis (Bigel.) Beck. Common on dry open soil.

Spirixtiles ceriol (L.) Richard. Oecasional in damp meadows.

Spimaxtimes Romaxzoffiaxa Cham. Frequent in wet fields and meadows.

Epipactis repens (T.) Crantz, var. ophioides (Fernald) A. A. Eaton. Woods, Sharks Point, Isle au Hant (N. T. Kidder).

Corallorritza maculata Raf. Rare. Rich moist woods, Dunham`s Point. Deer Isle (No. 2225).

*Corallomeinza trifida Chatelain. Isle an Hant (N. T. Fidder).

Microstruis viffolia (Michx.) BSP. Common in moist fields and meadows. Very abundant in damp depressions on the summit of Jerusalem Monntain, Tsle an Hant (No. $1719)$.

Tiparis Loeselin (J.) Richard. Rare. Swampy meadow, North Brooklin (No. 2906); *Isle an Hant (J. C. Arthmr).

\section{SALICACEAE}

Salix xigra Marsh. Rare. Near Brooklin Center (A. H. Graves).

Salix lecun Mruhl. Occasional. Swampy roadsides, Naskeag Point, Brooklin (No. 930); North Brooklin (No. $2907)$. 
Salix alba L., var. vitellixa (L.) Koch. Frequent along roadsides often oceurring spontaneously.

Saltx cordata Muhl. Rare. West Brooklin (A. H. Graves).

Silix miscolor: Mruhl. Common in moist open woods and fields.

Salix petiolaris Sm. Infrequent. Damp fields and roadsides. Brooklin and Swans Island.

Silix minilis Marsh. Frequent in wet ground.

Satix rostrata Richards. Common in fields and open woods.

Popelts alba L. Taturalized at Brooklin Center (A. H. Graves) : low ground, South Deer Isle (No. 1822).

Poptuts treatunines Michx. Almudant in elearings and dry open woods.

Poptles Graxtinextata Michx. Frequent in rich woods.

Poptris balshamera L. Occasional. Roadsides and low roods.

Popturs carincixs Ait. Infrequent. Escaped from eultiration. Brooklin and Swans Tsland.

Popeles neltomes Marsh. Rare. Escaped from cultivation. West Brooklin (A. H. Graves).

\section{MIYRICACEAE}

Mrrited Gate L. Common in sphagnum bogs and on pond margins.

Mrima carolixexsis Mill. Frequent in moist thickets and sterile soil along the shore.

Mrumca asplexifolia L.. Abundant in dry fields and on exposed hills. 


\section{BETULACEAE}

Coryuds rostrata Ait. Occasional in moist woods. Reach, Deer Isle (Nos. 1993, 2760).

Betula lutea Michx. f. Common in rich moist woods.

Betula popdlifolia Marsh. Abundant in clearings and open woods.

Betula alba L., var. Papyrifera (Marsh.) Spach. Common in woods.

rar. Cordifolia (Regel) Fernald. Frequent in woods.

Alxis crispa (Ait.) Pursh, var. aroluts Fernald. ${ }^{1}$ [Alnus mollis Fernald.] Abundant in moist woods, thickets and low ground. Also on sterile slopes and summits of hills. Ampes rachisa (T.) Moench. Frequent in low ground.

\section{FAGACEAE}

Fages graxpifotia Ehrh. Occasional in rich roods.

Quercus rubra L. Infrequent in rich soil.

var. ambigus (Michx. f.) Fernald. Infrequent. All fruiting specimens of the red oak were found to be this variety and it is possible that the typical form may be absent or very rare.

\section{URTICACEAE}

Ulmes americaxa L. Rare. Usually escaped from cultiration. Brooklin and Deer Isle.

Huxulus Lupulus L. Frequent in dry thickets along roadsides.

Urtica gracitis Ait. Occasional on barrier beaches.

1. Rhodora 15: 44 (1913). 


\section{LORANTHACEAE}

Arceuthobium pusillum Peck. Common on spruces. Donglas Cove, Isle an Hant. Infrequent elsewhere on the island (J. C. Arthmr', N. T. Kidder).

\section{POIYGONACEAE}

Ruarex Patientia L. Rare. Swampy meadow at head of Iong Pond, Isle an Hant (No. 1259) ; Rich's Cove, Isle an Taut (N. T. Kidder).

Romex Britaxica L. Rare. Wet roadsides, Brooklin (No. 1045); *Tsle an Haut (N. T. Kidder).

Rumex crispits L. Common in swamps and at edge of beaches.

Rumex patumes Bigel. Frequent on sandy or gravelly leaches.

Rrane Acetosella L. Abundant in dry fields and waste places.

Polygoxias Fowleri Robinson. Infrequent. Sandy beaches, Gray's Cove, Deer Isle (No. 2197); Swans Island (Miss Furbish, in Herb. N. E. Bot. Club).

Polygonua aviculare I. Tery common in waste places and ruderal ground. An extremely rariable species. A narrow leaved form (No. 657) from sandy beaches at Swans Tsland Head, Swans Island, closely approaches var. angustissimum Meisn.

var. vegetum Ledeb. Sandy beach, Swans Island Head, Swans Island (No. 691).

Polygondm allocarpiris Blake." Frequent on beaches.

1. Rhodora 2: 221-223 (1900).

2. Rhodora 19: 234-235 (1917). 
*Polggonear lapatififolicar L. Tsle au Hant (x. T. Kidder).

Polygonem tomextosum Sehrank. Common in fields, cultivated ground and along roadsides.

*Polycoxea Camert Olney. Isle au Hant (.J. C. Arthur, N. T. Kidder).

Polygoxum Hromopiper L. Abundant in swampy gromud.

Polygoxtan Persicaria L. Common in moist fields, along roadsides and in cultivated ground.

*Polycoxur mithopiperones Michx. Isle an Haut (N. T. Kidder).

Pomgoxuar sigititum L. Abundant in wet places.

Polygoxur Coxpouvus. Frequent in waste places and cultivated land.

Polyoxear crurone Michx. Almudant in dry elearings. var. enectum Peck. Road to village. Isle an Hant ( $\mathrm{X}$. T. Kidder).

Fagopyerar esculentex Moench. Occasional in fields and along roadsides. Swans Island and Deer Isle.

\section{CIIENOPODIACEAE}

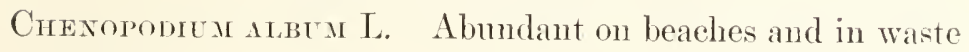
ground.

Atriplex patula L. Swans Island (Miss Furbish, in Herb. N. E. Bot. (lub).

var. mastata (L.) Gray. Abundant on beaches and in salt marshes.

Salicorita europatea L. Abundant on beaches and in salt marshes.

Suafda maritiana (L.) Dumort. Abundant on beaches of-ten below high tide level. 
Staeda Richir Fermald. Shores of Thoroughfare, Isle au Haut (N. T. Kidder).

Salsota Fild I. Occasional on dry sandy beaches.

\section{AMARANTHACEAE}

Ancuinterts retroflexus I. Rare. Cultivated ground, Brooklin (No. 1338).

Amamaxhus gramcizaxs L. Rare. Waste ground, Naskeag Point, Brooklin (No. 1337).

\section{CARYOPHYLLACEAE}

Spergularia rubra (L.) J. \& C. Presl. Frequent on sandy roadsides.

Spergelaria caxadexsis (Pers.) G. Don. Common on brackish shores.

Spergelaria salina J. \& C. Presl. ${ }^{1}$ Rare. Moist depressions in sandy beach, Gray's Core, Deer Isle (No. 1936).

Spergulatia leiosperata (Kindberg) F. Schmidt. ${ }^{2}$. Tnfrequent. Beach sands. Flyes Island, Brooklin (Nos. 594, 2989): Fish Creek, Deer Isle (No. 2803); Oceanville, Deer Isle (A. H. Norton); Trial Point, Isle an Hant ( . T. K. Kidder).

Sperctila arvexsis I. Abundant along roadsides and in cultivated ground.

Safixa pliocunbexs L. Frequent in springy places and on wet rocks.

1. Fl. vech. 95 (1819). See Fernald, M. L., and Wiegand, K. M., Rhodora 12: 162 (1910).

2. Reisen im Amurl. 131 (1868). See Fernald \& Wiegand 1. c. 162. 
Sagixa rodosa (L.) Fenzl, var. glandulosa (Bess.) Asch. Masons Ledge, near Heron Island, Swans Island (A. H. Norton); Little Spoon Island, Isle an Hant (X. T. Kidder).

Aremaria lateriflora L. Common in fields and on smmy banks near shore.

Arexarta peploides L., var. robesta Fernald. ${ }^{1}$ Occasional on sandy or pebbly shores, Brooklin and Swans Tsland; Fog Island, Isle an Hant (A. H. Norton). ${ }^{2}$

Arexarta Gromentaxtica (Retz.) Spreng. Oceanville, Deer Isle (A. H. Norton). ${ }^{2}$ Isle an Hant (N. T. Kidder).

Steldaita borealis Bigel., var. isopirylat Femald." Tnfrequent. Rich moist woods near serpentine quarry, North Deer Isle (Nos. 1950, 2222) ; Bradburys Island, Deer Isle (A. H. Norton); McGlatherys Tsland, Isle an Haut ( . T. Kidder).

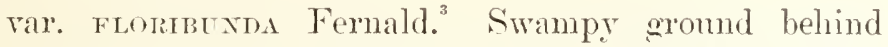
barrier beach, Red Point, Swans Island (No. 2S81): *Isle an Haut ( N. T. Kidder).

Steldalia memp (L.) Cyrill. Abundant in grassy places.

Cerastiral arverse L. Occasional. Grassy headlands near shore, Naskeag Point, Brooklin (Nos. 1415, 2997); Sandy Cove, Isle an Haut (N. T. Kidder); Spoon Ledge, Isle au Haut (A. H. Norton) ; *common at Isle au Haut (J. C. Arthmr).

Cerastiuar velatura L. Abundant along roadsides and in fields and waste places.

1. Rhodora 11: 114 (1909).

2. Rhodora 15: 139 (1913).

3. Rhodora 16: 150, 151 (1914). 
*Aghostemara Githago L. Oceasional. Fields and roadsides, Swans Island (Miss Kotz); Deer Isle (C. L. (urtis).

Silexe xoctiflora I. Rare. Fields, Flyes Island, Brooklin (Nos. .91, 2985); waste ground, South Deer Isle (No. 3067).

Sapoxiria officinalis L. Infrequent. Fields and roadsides, Brooklin and Deer Isle.

\section{PORTLLACACEAE}

Portulaca oleracea L. Rare. Weed in cultivated ground, Naskeag Point, Brooklin (No. 1363).

\section{NYMPHAEACEAE}

Nupinar variegatum Engelm. ${ }^{1}$ [Nymphaea advena Ait., var. carieguta (Engeln.) Fernald]. Common in ponds and bog holes.

Irmphafa onorata Ait. ${ }^{1}$ [Castuliu odorata (Ait.) Woodville \& Wrood.] Frequent in ponds.

\section{RANUNCULACEAE}

Raxuxculus Crmbalaria Pursh. Frequent on muddy shores and crevices of sea cliffs.

Ranunculus laxicaulis (T. \& G.) Darby. Rare. Muddy bog hole, South Deer Isle (Nos. 892, 1819). This species was reported by A. H. Norton from apparently the sane station. $^{2}$

Raxcicluts remexs L. Freguent in wet meadows and along streans. Brooklin and Swans Island.

1. For discussion of these changes in nomenclature, see Conard, H. S., Rhodora 18: 161-164 (1916).

2. Rhodora 15: 139 (1913). 
*Rixurcules bulboses L. South Deer Isle (A. H. Norton).

Raxuxceres acris 1. Abundant. Fields, roadsides and waste places. A specimen from H)eer Isle (No. 2081) has leaves which show all transitions from the trpical form to var. Sleveni, which would indicate that this variety might better be considered a form.

var. Stevexi (Andrz.) Lange. Oceasional. Meadows and pastures. Naskeag Point, Brooklin (No. 19sa).

Thabictrita polyganeas Miuhl. Common in wet gromed, often near shore.

var. nebecareum Fermald. Occasional in similar places.

Cumantis virginiaxa L. Rare. Roadsides near old eellar, Naskeag Point, Brooklin (No. 1:61).

Copts trifolia (L.) Salisb. Abundant in mossy woods. Aquilegia caxadexsis I. Rare. Dry bluff, Dumham's Point, Deer Isle (Nos. 2228, 2825).

Aquilegia vulgaris L. Infrequent. Eseaped from cultivation.

Actaea rubla (Ait.) Willd. Tare. Rich woorls near serpentine quarry, North Deer Lsle (Nos. 1952, 25.12).

\section{BERBERIDACEAE}

Bemberis velgais L. Tare. Established in field near old house, Naskeag Point, Brooklin (No. 2066i).

\section{PAPAVERACEAE}

Papareir Riroeas L. Rare. Waste ground near shore. Atlantic, Swans Island (No. 2329). 


\section{FUIIARTACEAE}

Anluana frygosa (Ait.) Greene. Rare. Roadsides, Naskeag Point, Brooklin (No. 2237). Eseaped from enltivation.

Cormtulis semperirexs (L.) Pers. Ocensional in dry clearings.

\section{CRTCIFERAE}

Lepidtur apetalda Wille. Waste ground near wharves, Stonington (No. 3065).

Lepintur campestre (L.) R. Br. Grassland near clubhouse, Point Lookout, Isle au Mant (N. T. Kidder).

Capseman Butra-pastolis (L.) Medic. Abundant in fields, waste places and along roadsides.

Canmelina sativa (L.) Crantz. Rare. A weed in cultivated ground, Naskeag Point, Brooklin (No. 1367).

Cakile mentrlat (Bigel.) Hook. Common on sea beaches.

Rapunas Rapifaxistruar L. Common in fields and waste places, often at edge of beaches.

Rupnuxts sutruts L. Oceasional as an escape from cultivation. Variable in color of flowers.

Brassica alivensis (T.) Kitze. Frequent in fields, pastures and waste places.

Brassica duxcea (L.) Cosson. Occasional. Waste ground, Swans Island (No. 2399): Isle an Haut (No. 1593).

Prasstea xigra (T.) Koch. Common in waste ground.

Brassica canpestris I. Rare. Cultivated fields, Naskeag Point, Brooklin (No. 251) ; gravelly roadsides, Atlantic, Swans Island (No. 2874).

(omingeia olinemtalis (L.) Dumort. Rare. Roadsides, Naskeag Point, Brooklin (No. 1798). 
Sismabrica officinale (L.) Scop. Rare. Sandy beach, Swans Island Head, Swans Island (No. 694).

- var. Leiocarpuar DC. Abundant in waste ground.

Erisimear cheiranthomes L. Oceasional. Tet meadow near shore, Flyes Island, Brooklin (To. 600); *Fog Island, Tsle au Haut (A. H. Norton).

Rimictia Armoracta (L.) Rolinson. Rare. Mroist fields, Atlantic, Swans Island (No. 1520).

Cardamine parviflora L. Rare. Dry soil on small island, Fish Creek, Deer Isle (No. 2801).

Cardanine pennsylvaxica Muhl. Tnfrequent. Tet roadside ditches, Naskeag Point, Brooklin (No. 1449); swampy meadows, Deer Isle (Nos. 2189, 2221).

Arabis Drumand Gray, var. conxexa (Greene) Fermald. Rare, Crow Nubble, Bradburys Island, Deer Isle ( 1. H. Norton ${ }^{1}$ in Herb. Port. Soc. Nat. Hist.). This station is the first in the eastern United States for this plant of western North Ameriea, although it oecurs in Têmiscoutata County, Quebec.

Amabis minstata (L.) Scop. Rare. Dry fields near shore, Flyes Island, Brooklin (No. 29s9a).

\section{SARRACENTACEAE}

Samirenta perperea L. Common in peaty swamps and sphagnum bogs.

\section{DROSERACEAE}

Drosera rotundfolis I. Alundant in swampy ground and sphagnum bogs.

Drosera loxgifolda L. Frequent in wet depressions in sphagnum bogs and borders of ponds.

1. Rhodora 15: 139 (1913). 


\section{CRASSULACEAE}

Sedrat acie L. Oceasional. Roeky ledges and roadsides. Escaped from cultivation.

Senea purpereun Tanseh. Infrequent. Meadows, head of Fish Creek, Deer Isle (No. 2833). *Isle au THant (J. C. Arthur).

Seir m mosertr (L.) Scop. Rare. Roeky shores near Duck Harbor, Isle an Hant (No. 1691). This aretie speeies is also very abundant on the sea eliffs of Placentia Island, two miles east of Swans Island, but has not been found on the latter island.

Semperifuma tectorum L. Oceasional in fields or waste ground. Escaped from cultivation.

\section{SAYTFRAGACEAE}

Rimes mintillux Michx. ${ }^{1}$ [Ribes oxyacanthoides L.] Commen in dry open woods and elearings.

var. Calcicola Fernald. Infrequent. Rich woods, Dunham's Point, Deer Isle (No. 2216); swampy woods, Sonth Deer Isle (No. 1835).

Ribes amertcaxum Mill. $\quad$ [Ribes floridum L'Her.] Rare. Near old house, Harrimans Point, Brooklin (A. H. Graves).

*Ribes lacistie (Pors.) Poir. Little Sheep Islind, southwest of Eagle Island Light, Deer lsle (1. H. Norton ${ }^{4}$ )]. This is the southern limit of this boreal speeies.

1. Fl. Bor. Am. 1: 111 (1803) ; see Coville, F. V., and Britton, N. L., N. A. Fl. 223 : 223, $225(1908)$.

2. Rhodora 13: 76 (1911).

3. Gard. Dict. ed. 8 No. 4 (1768); see Coville, F. V., and Britton, N. L., 1. c. 206.

4. Fhodora 15: 140 (1913). 
Ribes prostratuar L'Her. Common in low moist woods.

Ribes vulgare Lam. Rare. Fields near old house, Naskeag Point, Brooklin (No. 2065).

\section{HAMAMELIDACEAE}

Hamamelis vilgixiana L. Tnfrequent. Low moist woods and thickets.

\section{ROSACEAE}

Spiraea latifoli Borkh. Abundant in thickets and low ground.

Spiraea tomextosa L. Very eommon in open fields and moist ground.

Sorbarla sorbifolia (L.) A. Br. Occasional. Escaped from enltivation. Brooklin (No. 997); Swans Island (Nos. 771, 2877).

Prous Males L. Frequent. Eseaped from eultivation.

Pyies arbetifolia (L.) I.f., var. atroptrpurea (Britton)

Rohinson. Common in wet gromd and frequent on headlands.

Prous melaxocarpa (Michx.) Willd. Frequent in low ground and on the barren slopes and summits of hills.

Prots anericana (Marsh.) DC. Common in moist woods. *Prnus sitcuexsis (Roem.) Piper. Tsle an Hant (J. C. Arthur).

*Anelaxchier canalexsis (T.) Medic. Isle an Mant (J. C. Mrthur); Noores Harbor, Isle an Hant (N. T. Kidder). In Graves "Woody Plants of Brooklin, Maine," the anthor eites Amelanchier canadensis as being common. The specimens from this loeality in the herharim of Yale University, however, are hrbrids between A. laevis and some other species.

1. Rhodora 12: 180 (1910). 
Amemaxcher Laevis Tiegand. ${ }^{1}$ Common in moist woods and swampy thickets. This species is very variable and hybridizes freely with the other species in the region. The typical form is well shown in No. 1783, swampy woods, Sedgwick road. Brooklin; and Nos. 1480, 2507, damp cool wonds, Mackerel Core. Swans Island. The following hrorrids have been found:-

A. laevis $\times$ A. oblongifolia. Swampy roadsides, Gray's Core, Deer Isle (No. 2087) ; moist thickets near shore, Reach, Deer Isle (Tos. 2095, 2099). The first two of these speeimens "are almost pure $A$. luevis, while the last seems to show a little more $A$. oblongifolia tendener."

A. Lanvis $X$ a hairy leaved species. Swampy roods near Lily Pond, South Deer Isle (No. 1807). "This hybrid," to quote Wiegand, "certainly has the blood of 1. laeris in it, but there is evidence of some other blood of hairy leaved species as the leares are, when mature. somewhat hairy beneath. The only three possibilities are 1 . oblongifolia, A. stolonifera and $A$. canadensis." Rocky woods on slopes of Sawyers Mt., Isle an Hant (No. 1700). This hybrid shows a similar condition "except that the shape of the leaf suggests A. oblongifolia or A. stolonifera and not A. canudensis."

1. Lafis $\times$ 1. Bimpramiax. Cool moist woods near head of Long Pond, Isle au Haut (No. 1614).

1. Lueris $X$ (?). Cool moist woods, Goose Pond, Swans Island (No. 1614): Brooklin (1. H. Graves).

1. Rhodora 14: 154 (1912). 
*Amelaxcher Bartramiana (Tansch.) Roem. ${ }^{1}$ Flye Point, Brooklin (A. H. Grares). In his list of Brooklin plants Graves inchudes A. oligocarpa (Michx.) Rocm. with the remarks, "Tather common. Flye Point. Tr. IV. H. Blanchard, to whom I have referred the specimens, states that it is probably this species." Wiegand cites $A$. oligocarpa as a symonym of 1. Bartramiana. The following hrbrids of A. Bartramiana have been collected:A. Barthamata $\times$ 1. Laevis (see under 1. Taevis). A. Bartramiana $\times$ A. oblongifolia. Rich woods near Mackerel Core, Swans Tsland (No. 1475).

Cratafers rotradifolia Mench. Open thickets near shore, Gray's Cove, Deer Isle (Nos. 1958, 1959).

Crataegus Jonesae Sargent. Roadsides. Naskeag Point, Brooklin (Nos. 1026, 2070) : rare, Isle an Hant village (J. C. Arthnr).

Cliatafeirs macrosperama Ashe, approaching var. acutiloba (Sarg.) Eggeleston. Rich woods, Oak Point, Reach, Deer Isle (No. 21:31).

Cratafgres machacantila Lodd. Frequent in fields, open woods and thickets near the shore.

Fragaria virgivaxa Dnehesne. Abundant in fields and meadows.

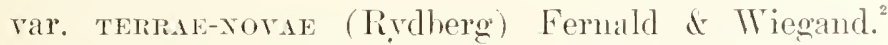
Dry open places in woods, Reach, Theer Isle (No. $2752 a)$.

Potextilia moxspenexsis L. Frequent in roadsides and fields.

rar. xorvecica (L.) Ridb. Damp roadsides near Moore's Harbor, Isle au Haut (No. 1160).

1. Syn. Rosif. p. 145. (1847); see Wiegand, K. M., Rhodora 14:158 (1912).

2. Rhodora 13: 106 (1911). 


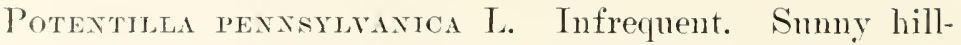
side, Naskeag Point, Brooklin (No. 3019); open fields near serpentine (narry, Reach, Deer Isle (No. 2142); headlands, Dunlam's Point. Deer Isle (No. 2232); *near village, Isle an Hant (J. C. Arthur, N. T. Kidder).

Potextilar arentea L. Frequent on dry sterile soil.

Potentilla palustris (L.) Scop., var. villosa (Pers.) Lehm. Tery common on hoggy shore of Torrey Pond, Deer Isle (No. 2183); Merehants Island, Isle an Haut ( . T. Kidder).

Potextulla trinextata Ait. Common in sterile fields on headlands and exposed hilltops.

Potentilita pacifica Howell." (Potentilla Anserina I.) Abundant on shores and in salt marshes.

Potextilla canamensis L., var. smplex (Michx.) T. \& G. Very common in dry fields, roadsides and waste places.

Germ rivale L. Occasional in wet meadows. Naskeag Point, Brooklin (No. 1450); North Brooklin (No. 2912); South Deer Isle (No. 1826).

Rubrs maers L., rar. caxnuexsis Richards. ${ }^{3}$ [var, amleatissimus (C. A. Mey.) Regel \& Tiling.] Frequent in thickets and fields and very abundant in clearings.

Rubus pubescexs Raf.' [Rubus triflorus Richards.] Common in swampy woods.

1. Stirp. Pug. 9: 44 (1851). See Fernald, M. L., and Long, B., Rhodora 16: 5-11 (1914).

2. Fl. N. W. Am. 1: 179 (1898). See Robinson, B. L., and Fernald, M. L., Rhodora 11: 48 (1909).

3. Frankl. Jour. 739 (823). See Blanchard, W. H., Rhodora 11: 236 (1909).

4. Med. Repos. N. Y. Ser. III, 2: 333 (1811). See Blanhard 1. c. 
var. Pilosifolies A. F. Hill. ${ }^{1}$ Occasional in wet woods.

Rubus aldenilaxiexsis Porter. "Common at Brooklin (A. H. Graves): Tsle an Hant (.T. C. Arthur in Herb. Gray). Hrbrids of this species, nnder the name $R$. nigrobaccus Bailer, have been cited by Rydberg, based on material from Isle an Hant: $R$. nigrobaccus $\times R$. nitgricans (.T. C. Arthur, No. .22); R. nigrobaccus $\times$ R. pergratus (J. C. Arthur, Nos. 5.5, .56); R. nigrobaceus $\times$ R. amicalis (.T. C. Arthur).

Rumes percratrs Blanchard. Frequent in moist elearings and thickets. Deer Tsle and Tsle an Hant. The following hybrids occur at Isle an Haut: $:^{3} R$. pergratus $\times R$. umicalis (.T. C. Arthur, No.53); R. pergratus $\times$ R. nigrobaccus (see mder R. alleghaniensis).

*Rebus elefintriles Blanchard. Tsle an Hant (.T. C. Arthur, No. 51), determined by Rydberg."

Rubes canadexsis L. Occasional in fields and pastures. This species inchudes $R$. amiculis Blanchard, cited from Tsle au Hant by Rydberg" (.T. C. Arthur, Nos. 50, 58). Rubus recrlivicarlis Blanchard. Rocky pasture, Babson's Ridge, Brooklin (No. 112 ).

Rumes mspmes L. Very common in fiekls and low ground. Dalibarda repexs L. Tnfrequent. Wet mossy woods, Naskeag Point, Brooklin (No. 657); Atlantic, Swans Island (No. 2274) ; Reach, Deer Isle (No. 2776).

1. Rhodora 16: 152 (1914).

2. N. A. Fl. 225 463 (1913). See also Bull. Torr. Bot. Club. 42: 468 (1915).

3. Rydberg, 1. c.

4. Bull. Torr. Bot. Club 42: 153, 154 (1915). 
Agrimoxta griposepala Wallr. Moist thickets in woods, Gray's Cove, Reach, Deer Isle (No. 2866).

Rosa spixosissma T. Naturalized at Flye Point and Harriman Point, Brooklin (A. H. Grares).

Rosa cixranomea L. Naturalized at North Brooklin (A. H. Graves).

Rosi rubignosi L. Pastmes, Naskeag Point, Brooklin (ג. H. Graves).

Rosa vitida Willd. Common in bogs. Swans Island and Isle an Hant.

Rosa virefinana Mill. Very eommon in swamps and roadside thickets.

Rosa indmuis Marsh. Abundant. Dry fields and roadsides.

Pruxus serotrixa Ehrh. Rare and loeal. Roadsides, North Brooklin (Nos. 2443, 2921); rieh upland woods, Deer Isle (No. 2573).

Proxis virgintana $\mathrm{L}$. Common in woods and thiekets.

Proxus penserluarica L. f. Common in dry open woods and elearings.

Prun es Aviun L. Naturalized near roadside about one-half mile north of Haven, Brooklin (A. H. Graves).

\section{LEGUMINOSAE}

Trefolutu arvexse L. Frequent in fields and along roadsides.

Trifolium pratexse T. Abundant in meadows and fields.

Trifoliua repens L. Common along roadsides and in fields.

Trifolium myerioum L. Frequent in fields and meadows. 
Trifolium agrarium L. Common in fields and along roadsides.

Trifolium procumbexs I. Very common along roadsides.

Melilotus alba Desr. Moist roadsides near Haven, Brooklin.

Robinia Pseudo-Acacia L. Occasional. Naturalized in fields and roadsides.

Robinia viscosa Vent. Established near roadside at Flye Point, Brooklin (A. H. Graves).

Vicia angustifolia (I.) Reichard, var. segetalis (Thuillier) Koch. Frequent at edge of beaches, in waste ground and cultivated fields. Brooklin and Deer Isle.

Vicia Cracca L. Occasional. Fields and meadows.

Lathyrus maritmus (L.) Bigel. Very common on beaches and shores. Occasional inland, introduced with sea weed for fertilizer.

var. Glaber (Seringe) Eames. ${ }^{1}$ Common on beaches.

Lathyrus palustris L., var.pilosus (Cham.) Ledeb. Common in wet meadows, especially near the shore. A very variable plant with leaves often near the var. macranthus (T. C. White) Fernald.

Amphicarpa monoica (L.) Ell. Damp open places in woods, Gray's Cove, Reach, Deer Isle (No. 2864).

\section{IINACEAE}

Linum usitatissimua L. Gravelly roadsides near Long Pond, Isle au Haut (No. 3061).

1. Rhodora 11: 95 (1909).

2. Rhodora 13: 50 (1911). 


\section{OXALIDACEAE}

Oxalts americaxa Bigel. ${ }^{1}$ [Oxalis Acetosella of Gray's Mannal, Ed. 7.] Common in cool mossy woods.

Oxalis corxiculata T. Abundant in fields and along roadsides.

\section{GERANTCEAE}

Geraxiear Robertiaxum T. Occasional on barrier beaches. North Point, Swans Island (No. 1464); Hockomock Head, Swans Island (No. 2333); Isle au Haut (J. C. Arthur, A. H. Norton).

Geraxim Bickxelum Britton. Infrequent. Waste ground, Gray's Cove, Deer Isle (No. 2527); roadsides, Isle au Hant (No. 1607).

\section{POL, YGALACEAE}

Polygala saxgurea L. Rare. Fields near Gray's Cove, Deer Isle (No. 1920).

Polygala vemtrcillata L. Infrequent. Fields and meadows, Reach, Deer Isle (No. 191S); North Brooklin (No. 2913).

\section{ETPHORBTACEAE}

Erphorbia Crparissias L. Common. Roadsides and waste places.

\section{CAJLITRICHACEAE}

Callitriche paldstis L. Quiet waters of Torrey Pond, Deer Isle (C. L. Curtis).

1. Fl. Bost. Ed. 2: 258 (1824). See Fernald, M. L., Rhodora 20:76 (1918). 


\section{EMPETRACEAE}

Empetruar xigrear L. Common on headlands and barren outerops.

Corema Coxrabit Torr. Frequent. Sterile outerops and exposed summits of hills. Selist outcrops, Central Tract, Brooklin (Nos. 105.3, 3012; also A. H. Graves); barren granite hills, Swans Island Head (No. 795 ) ; barren summit of Mt. Champlain, Isle au Haut (No. 1299). Some thirty years ago Corema was considered a great rarity in our flora and new stations for it were frequently published. Among them were two from the Penobscot Bay region. Tohn H. Redfield writes ${ }^{1}$ that Chas. S. Wilder found the plant on Deer Isle, "not far from Green's Landing (Stonington) on the right of the road leading to North West Harbor, on a rocky eminence in a small pasture." The same authority cites several stations on Isle an Hant, "the barren summit of the island," "Black Dinah," a hare, round roeky knoh near Moores Harbor and one or two other localities.

\section{ANACARDTACEAE}

Rhus trphisa L. Infrequent. Dry fields and hillsides.

Rinus Toxicodendiox L. Oceasional on headlands or in dry fields near the shore.

\section{AQUIFOLIACEAE}

Ilex verticillata (T.) Gray. Common in moist woods and roadside thickets.

var. Padifolia (Willd.) T. \& G. Frequent in similar. habitats. The specimens referred by Graves to var. tenuifolia (Torr.) Wats. belong to this variety or to the type.

1. Bull. Torr. Bot. Club 13: 221 (1886).

2. Bull. Torr. Bot. Club 11: 99 (1884). 
Nemopanthus mucronata (L.) Trel. Abundant in swampy woods.

\section{ACERACEAE}

Acer pennsiluanicuar L. Common in moist woods.

Acer spicatum Lam. Common in moist woods.

Acer saccharum Marsh. Rare. Woods near Haven, Brooklin (A. H. Graves); Isle au Haut (N. T. Kidder). Acer rubrum L. Frequent in swampy woods.

\section{BALSAMTNACEAE}

Tmpatiexs biflora Walt. Abundant in wet places, especially near the shore.

- VITACEAE

Psemera quixquefolia (L.) Greene. Established at old house site, Harriman's Point, Brooklin (A. H. Graves).

\section{MATVACEAE}

MALVA RotundfFolia L. Occasional about dwellings.

\section{HYPERICACEAE}

Hypericum perforatum L. Common in dry fields and along roadsides.

Hypericum boreale (Britton) Bicknell. Isle au Haut (Miss Kate Furbish, in Herb. N. E. Bot. Club.)

Hrpericuar antilua L. Frequent in low grome.

Hypericta majes (Gray) Britton. Occasional in muddy roadside ditches, North Brooklin (No. 2920); Swans Island (Nos. 2352, 2698); Isle au Haut (No. 1757). Forms, apparently hybrids between this species and the following, sometimes occur. Muddy roadsides, Isle au Haut (No. 1757a). 
Hypericuar caxadexse L. Common in wet soil.

Hypericuar virgixicua L. Abundant in swampy ground.

\section{CISTACEAE}

Hrisonla ericoides L. Summit of Sawyer's Mountain, Isle an Hant (N. T. Kidder).

Lechea isternedia Leggett. Common in dry sterile soil. var. Juxtperta (Bicknell) Robinson. Occasional along roadsides.

\section{VTOLACEAE}

Viola cucullata Ait. Common in wet meadows, moist woods and swampy ground. Forma PRIONosepala (Greene) Brainerd. ${ }^{1}$ Occasional. Rich woods near Gray's Cove, Deer Isle (No. 2127) ; moist roadsides, Isle au Hant (No. 1641).

Viola septextrioxalis Greene. Infrequent. Open woods on serpentine, Little Deer Isle (Nos. 2582, 25S4); rich mossy woods near serpentine quarry, Reach, Deer Isle (No. 2619); Tsle an Hant (N. T. Kidder).

Viola fimbriatula Sm. Rare. Dry headlands, Dumham's Point, Deer Isle (No. 2229).

"Viola primilifolia L. Isle an Haut (J. C. Arthur). fields.

Viola pallexs (Banks) Brainerd. Frequent in wet ground.

Viola mcognita Brainerd. Abundant in damp mossy woods.

1. Rhodora 15: 112 (1913). 
var. Forbesil Brainerd. ${ }^{1}$ Rich woorls near Torrey Pond, Deer Isle (No. 198s) ; wet mossy woods, Dunham's Point, Deer Isle (No. 2227); cool moist woods, Atlantic, Swans Island (No. 2277).

Viola coxspersa Reichenb. Rare. Rich woods near serpentine quarry, Reach, Deer Isle (Nos. 2604, 2620).

\section{I.YTHRACEAE}

Decodon vertichlatits (L.) Ell., var. hanigates T. \& $\mathrm{G}^{2}$ Occasional. Swampy margin of Torrey Pond, Deer Isle (No. 1969) ; edge of sphagnmm mat in bog near Rich's Core, Isle au Haut (No. 1197; also J. C. Arthur).

\section{ONAGRACEAE}

Epilobica axgustifolien L. Common in open fields and clearings, especially in reeently burned ground.

Epilomin molle Torr. Frequent in wet swampy meadows. Epilobicar denstar Raf. Common in swamps and wet ground.

Epilobica paleste I. Swans Island (Miss Furbish, in Herb. N. E. Bot. (lub).

var. vroxticota Hanssk. Frequent in sphagmum bogs. Epilobiux grandulosuar Lehm., var. adenocaulon (Hanssk.) Fernald. ${ }^{3}$ [Epilobium adenocaulon Hanssk.] Very common in low ground, especially near the shore. A deparperate form of this variety, collected in springy places in a field on Hat Island, Swans Island (No. 106), was wrongly attributed in an earlier paper ${ }^{4}$ to E. adenocanlon, var. perplexans Trel.

1. Bull. Torr. Bot. Club 38: 8 (1911).

2. Fl. No. Am. 1: 483 (1840). See Fernald, II. L., Rhodora 19: 154 (1917).

3. Rhodora 20: 35 (1918).

4. Rhodora 16: 192 (1914). 
Oenothera muricata L. Very common on shores, in dry fields and along roadsides.

Oenothera pumla L. Common in dry fields and pastures. Circaea alpixa L. Common in wet mossy woods.

\section{ARALTACEAE}

Aralia racemosa L. Rare. Rieh woods, South Deer Isle (No. 1842).

Aratra mispina Vent. Common in elearings and on roeky open soil.

Aralia xudicarlis T. Frequent in woods.

\section{UMBELLIFERAE}

Sanicula malilandica L. Rare. Dry open woods near Gray's Cove, Deer Isle (Nos. 2188, 25:36).

Hyorocotyle americana T. Oceasional. Springy places in fields and meadows.

Conica maculatum L. Waste ground, the Valley, Hockomoek Head, Swans Island (No. 2666).

Cicuta maculata L. Common in wetground.

Ciclta bulbifera L. Rare. Swampy margin of Lily Pond, South Deer Isle (No. 1849).

Carua Carvi I. Very common along roadsides and in drey fields and waste places.

Siuar cicutaefolium Sehrank. Rare. Muddy shores of Torrey Pond, Deer Isle (Nos. 19S6, 2856).

Zizia aurea (L.) Koeh. Rare. Meadow near Gray's Cove, Deer Isle (Nos. 2110, 2545).

Ligusticum scothicun L. Common on rocky shores and beaches, oceasionally at edge of salt marshes. 
Coelopleulua actaeifolica (Michx.) Coult. \& Rose. Common on rocky shores and headlands on the outer islands, Swans Island and Isle au Haut, and occasionally on shores inland.

Pastixaca sativa L. Occasional. Wet swampy meadow, Flyes Point, Brooklin (Nos. 962, 2969; meadows near shore, Naskeag Point, Prooklin (No. 204).

Heracteum Laxatru Michx. Frequent on eroding bluffs along shore. Occasional in low marsly ground.

Congosemixum cunnexse (L.) BSP. Moist thickets in rich woods, Gray's Cove, Reach, Deer Isle (No. 2868).

Datcr's Carota. Occasional in fields and meadows, Brooklin (Nos. 2451, 3005).

\section{CORNACEAE}

Corses caxadexsis L. Common in wonds and open places. var. 1xтendedia Farr. ${ }^{1}$ Very rare. Dry sterile slopes of Mt. Champlain, Isle an Haut (No. 1287). This is the first station in the East outside of Labrador and western Newfoundland for this variety. ${ }^{2}$

Corses circinata L'Her. Infrequent. Wet banks of stream in rich woods, Atlantic, Swans Island (No. 2397).

Corxes stoloxifera Michx. Rare. Low wet woods near Rerl Point, Swans Island (No. 2885).

Corves alternifolia L. f. Common in thickets and open woods.

\section{ERTCACEAE}

MIoxeses criflora (L.) Gray. Frequent in damp mossy woods.

1. Contrib. Bot. Lab. Univ. Penn. 2: 423 (1904).

2. See Fernald, M. L., and Wiegand, K. M., Rhodora 13: 107 (1911). 
Prrola seccrda L. Rich mossy woods, Naskeag Point, Brooklin (No. 2976).

var. oвtusata Turcz. Rare. Rich woods near serpentine quarry, Reach, Deer Isle (No. 2667).

Pyrola chloraxtha Sw. Rare. Rich open woods, Gray's Core, Reach, Deer Isle (No. 2796).

Prrola elliptica Nutt. Common in dry open woods.

Prrola axericana Sweet. Infrequent. Dry open woods, Naskeag Point, Brooklin (No. 654); dry meadow, North Point, Atlantic, Swans Island (No. 2246).

Moxotropa uniflora L. Common in rich woods.

Lenex groentandictar Oeder. Common in sphagnum bogs and moist thickets.

Rhododexdron caxadexse (L.) BSP. Common in swampy woods and bogs.

Kalina axgestifolia L. Abundant in dry open ground and occasional in bogs.

Kalmia polifolia Wang. Frequent in sphagmum bogs.

Chamaedapine calycilata (T.) Moench. Common in bogs and swampy pond margins.

Gavltheria procumbexs L. Common in dry woods and open ground.

Arctostaphylos Uva-urisi (I.) Spreng. Oceasional. Isle an Haut; barren hillside near Juck Harbor (No. 1749); headlands at the Cliffs (No. 3054).

Chlogenes hispidela (L.) T. d G. Common in damp mossy woods.

Gaylessacia demosa (Andr.) T. \& G., var. Bigeloviana Fernald." Occasional in sphagnum bogs. Stockbridge Pond, Swans Island (No. 2:20); Marsh Cove, Kimball's Island, Isle an Hant (No. 1662).

1. Rhodora 13: 99 (1911). 
Gaylussacia baccata (Tang.) C. Koch. Common in swampy thickets and open ground.

Vacomiua pennsyluanicum Lam. Very common in dry open ground and on sterile mountain tops.

var. vigrua Wood. Occasional in dry fields.

Vaccisiear caxamexse Kalm. Common on dry or moist ground.

Thacinitur cormanosex L. Rare. Swampy woods near Lily Pond, South Deer Isle (No. 1816).

Vaccinius atrococoum (Gray) Heller. Occasional. Sphagnum bog near Sedgwick road, Brooklin (Nos. 1059, 1771; swampy woods near north end of Herricks Bay, Brooklin (A. H. Graves). This plant was referred in "Woody Plants of Brooklin" to V. corymbosum; swampy shores of Torrey Pond, Deer Isle (No. 1961).

Vaccinium Vitis-Thaea L., var.uinus Lodd. Common in dry fields and on sterile slopes and summits of hills.

VAccinfun Oxrcoceus I. Occasional in sphagnum bogs.

VACCiNium Macrocarpox Ait. Common in bogs and swampy gromid.

\section{PLIIIBAGLNACEAE}

Limonida trichogonum Blake." [Limonium carolinianum of Grar's Manual, Ed. ־.] Abundant on muddy and rocky shores and in salt marshes.

\section{PRIAIULACEAE}

"Frsmagina vulgaris L. Isle au Haut (J. C. Arthur).

Lrsmichia QUADrifolia L. Frequent. Dry fields and roadsides.

1. Rhodora 18: 61, 62 (1916). 
X Lysmachia probecta (Gray) Fernald. Rare. Muddy roadside ditches, Isle an Haut (No. 1612).

Lysmacina terrestris (I.) BSP. Common in swampy ground.

Triextalis borealis Raf. ${ }^{1} \quad$ [Trientalis americana (Per's.) Pursh.] Frequent in low woods.

Glatx martina L. Pebbly beach, Goose Island, Blue Hill Bay, Brooklin (No. 10s); rare, Isle an Haut (N. T. Kidder).

var. obtusifolia Fernald. Common on shores and in salt marshes.

Axagallis arvensis T. Infrequent. Mroist fields and pactures, South Deer Isle (No. 1806); headlands, Durham's Point, Deer Tsle (No. 2828).

\section{OLEICEIE}

Fraxixes americana L. Frequent in moist woods.

Fraxines pexpspluarica Marsh. Oceasional in rich damp woods. Deer Isle and Isle an Haut.

Fraxixts xigra Marsh. Infrequent. Wet places in ('entral Tract, Brooklin (A. II. Graves); swampy woods, South Deer Isle (No. 18:32).

Syrixga veliaris L. Occasional in woods, eseaped from enltivation.

\section{GENTIANACEAE}

Halexta deflexa (Sm.) Griseb. Rare. Wet meadows, Naskeag Point, Brooklin (No. 2235); Brooklin (C. E. Faxon, in Herb. Gray).

1. Med. Repos. N. York Ser. II: 5354 (1808). See Blanchard, IV. H., Rhodora 11: 236 (1909). 
Mienyanthes trifoliata L. Rare. Swampy woods, South Deer Isle (No. 1\$24).

Nrmpioides lacurosum (Vent.) Fernald. Infrequent. Goose Pond, Swans Island (No. 2376).

\section{APOCYNACEAE}

Apocymen aximosamamfolium L. Frequent in dry fields and thickets.

\section{CONVOLVULACEAE}

*Coxvolvulus sepiuar I. Isle au Haut (N. T. Kidder). var. pubescens (Gray) Fernald. Common at edge of gravelly beaches.

Cuscuta Grovori Willd. Common in wet places.

\section{HYDROPHYILACEAE}

Phacelia linearis (Pursh.) Holz. ${ }^{1}$ Rare. A single plant was found in a barn yard at Tamarack Lodge, Brooklin (No. 2514), probably introduced from the West in grain. This is the first station for the plant in Maine and the second in New England. Mr. C. A. Weatherby collected it in a sandy field at Windsor, Conn.

\section{BORAGINACEAE}

Mertensia maritiala (L.) S. F. Gray. Common on barrier beaches and rockr shores on the outer islands.

\section{LABIA'TAE}

*Teucriuar caxadexse L., var. littorale (Bicknell) Fernald. Isle an Haut (N. T. Kidder).

1. Contrib. Nat. Herb, 3: 242 (1\$95). 
Teuchum occinextale Gray, var. Borenle (Bicknell) Fernald. Occasional. Moist ground at edge of beaches, Flyes Point, Brooklin (No. 947); Fish Creek, Deer Isle No. 2804); shingle beach, Stanley Point, Minturn, Swans Island (No. 2932).

Scutellaria latermiola I. Infrequent. Low wet woods near Horrick's Bay, Brooklin (No. 1091); swampy woods near Lily Pond, Sonth Deer Isle (No. 1804).

Scutellaria calemiculati T. Abundant at edge of beaches and in moist ground near the shore.

Nepeta Cataria L. Occasional along roadsides and near houses.

Nepeta inederacea (J.) Trevisan. Infrequent. Roadsides near houses, Naskeag Point, Brooklin.

Pidunella vulgaris L., var. lanceolata (Barton) Fernald. ${ }^{1}$ Abundant in fields and meadows. Forma CANDIDA Fernald. Moist roadsides, Naskeag Point, Brooklin (No. 2241). Forma ionocalix Femald. Very common in dry fields and along roadsides.

Galeopsis Tetrahit L., var. bifida (Boem.) Lejeune \& Courtoir. ${ }^{2}$ Very common in waste ground, along roadsides and in cultivated land.

Leonerus Carmiaca L. Occasional in waste ground and about houses.

Heneoma pulegiones (L.) Pers. Common in dry fields and pastures.

Lxcopus uniflones Michx. Very common in moist ground. Lycopes anericaves Muhl. Common in wet places.

1. Rhodora 15: 183, 184 (1913).

2. Compend. Fl. Belg. 2: 241 (1831). See Fernald, M. L., and Wiegand, K. M., Rhodora 12: 142 (1910). 
* Mextila piperita L. Barred Harbor, Isle au Hant (N. T. Kidder).

\#Iextua gentuis L. Isle an Hant (J. C. Arthur).

Mextira arvexsis L. Tnfrequent in moist ground.

var. Caxamensis (L.) Bripuet. Very eommon in wet places, especially near the shore.

\section{SOLANACEAE}

Peturia violacea Lindl. Rare, when not under enltivation. Gravelly beach, Atlantic, Swans Island (No. 2330).

Solaxiar Delcamara L. Tnfrequent. Fields and roadsides, Reach, Deer Isle (No. 2141) : moist elearings near Torrey Pond, Deer Isle (No. 25.51).

Solanim ximsin L. Rare. Wet ground near shore, Marsh Cove, Kimball's Island. Isle au Haut (No. 16.7); * sea beaches, Tsle an Hant (.T. C. Arthur).

\section{SCROPHULARTACEAE}

Terbascly Thapsis I. Common in fields and pastures.

Timaria velgaris Hill. Oeeasional in fields and elearings. Naskeag Point, Brooklin (No. 1119); North Brooklin (No. 2896) ; Atlantie, Swans Tsland (No. 2263).

Linaria Caxamensis (L.) Dumont. Common in dry fields and sandy soil.

Chelone ribara L. Frequent in wet gromud.

Veroxica anericaxa Sehwein. Rare. Swampy margin of lrook, Little Deer Isle (No. 2589).

Veroxica scutellata L. Infrequent in wet places. Swampy meadow, Gray's ('ove, Deer Isle (No. 2568).

Teronica serpyltifolia T. Rave. Sumy hillside, tittle Deer Tsle (No. 2.9.5) : Isle an Mant (N. T. Kidder). 
Teroxich peregrits. L. Occasional in dry soil and on headlands, Black Point, Swans Tsland (No. 2340): small island in Fislı Creek, Deer Isle (No. 2802).

Vernxica arvexsis T. Common in dry fields, especially near the shore.

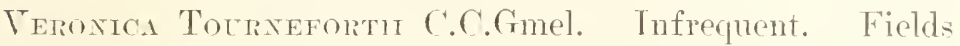
and roadsides, Garden Point. Swans Island (No. 2702); Isle an Mant (.J. C. Arthmr).

Melanpytan mixeare Lan. Common in dry fields and clearings.

Ftrumasia purpltea Reeks, var. Raxterj (Robinson) Fernald of Wiegand. ${ }^{1}$ [Euphrusia Rundii Rohinson.] Common on dry headlands on the outer islands. Black Point, Swans Tsland (No. 29:92): Hoekomock Head. Swans Tsland (No. 2669); Isle an Hant (. . T. Kidder). Forma Albifure Fernald \& Miegand. Pare. Dry fiedd. near shore. Intrek Harlor, Is]e an Haut (No. 1750).

Evpmasia striota Host. ${ }^{2}$ Frepment in dry fields. Atlantic. Swans Island (No, 3080) : Swans Island (Miss Furbish, in Merh. X. E. Bot. ('lub): fields and roadsides. Sunshine, Teer Isle ( No. 2!4).

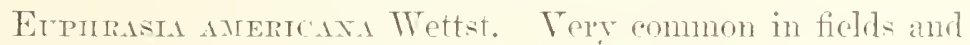
pastmres and along roadsides.

Opoxtites rébra Gilib. Freouent in dry fields and along roadsides.

Pencratas cuxanexsis L. Frequent in fields and dry opent woods.

Rinichathes Consta-galdi L. Tery common. Fields, poadsides anil near dwellings.

1. Rhodora 17: 188 (1915).

2. Fl. Austr. 2: 185 (1831). See Fernald, M. L., and Wiegand, K. II., Rhodora 17: 197 (1915). 


\section{LENTIBULARLACE.AE}

Utiotelania velgaris L., var. anericaxa Grag. Frequent in pond holes in sphagmm logs.

Utricularia ixterineisa Harne. Occasional in muddy ponds. Stockbridge Pond. Swans Tsland (No. 2322); Goose Pond, Swans Island (No. 2682).

Ttriclearia corxeta Michx. Occasional in wetter portions of sphagnum bogs. Stockbridge Pond bog. Swans Island (No. 2317) ; bogery shores of Sadler Pond, Minturn. Swans Island (No. 2670 ).

\section{OROBANCHACEAE}

Epifages ripenviaxa L. Rich woods near Lily Pond, South Teer Isle (No. 3072 ).

Orobaxche rififoma L. Rich moist woods, Gray's Cove, Reach, Deer 1sle (No. 27\%t).

\section{PJANTAGTNACEAE}

Plaxtago major L. Very common. Fields and roadsides. var. ixternenia (Gilibert) Dene. Rare. Sandy beach. Greenlaw's Neck, Deer Isle (No. 2811).

Plaxtano neciplexs Barneond. Abundant on beaches, rocky shores and in salt marshes.

Plaxtaco lanceolata L. Rare. Gravelly sloore of Mill Pond, Deer Isle (No. 2214).

\section{RLPIACEAE}

Galium Aparise L. Infrequent. Edge of peblly beach. North Point. Swans Island (No. 2434); edge of beach, Barred Harbor, Isle an ITant (N. T. Kidder). *(Galitu veruar L. Isle an Haut (N. T. Kidder). 
Galium palustre L. Occasional in wet meadows near head of Mackerel Cove, Swans Island (No. 1t71); Gray's Cove, Deer Isle (No. 254:3).

Gatidur trifidun T. Frequent in wet mossy woods and low ground.

Galiuar Chaptoxi Michx. Very common in swampe meadows.

Galtua asprelum Michx. Rare. Moist thickets near Torrey Pond, Deer Isle (No. 1975); damp thickets. Reach, Deer Isle (No. 2775).

Galitar trifloruar Michx. Common in meadows and wet mossy woods.

Mitchelda repexs L. Therequent in rieh woods. Near Merrick's Bay, Brooklin (No. 2995); Atlantic, Swans Fsland (No. 2659); Isle an Haut (N. T. Kidder).

Horstoxia caeriten L. Common in moist fields and meadows.

\section{CAPRIEOLIACEAE}

Diervilla Loxicera Mill. Common in roadside thickets and dry woods.

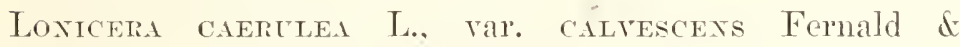
Wiegand. ${ }^{1} \quad$ Frequent in sphaguum logs.

rar. viluosı (Michx.) T. \& G. Tnfrequent. Shores of Herrick's Bay, Brooklin (No. 2973): damp woods near Mackerel Cove, Swans Island (No. 14t t): boggy meadow, outlet of Croose Pond. Swans Island (Nos. 1490, 2673).

Loxicema caranexsis Marsh. Common in rich moist woods.

Loxicera sempervinexs L. Established near roadside, Flyes Point, Brooklin ( 1 . H. Graves).

1. Rhodora 12: 210 (1910). 
Sirarphoricarpos albes (L.) Blake, var. chevigatus (Fermald) Blake. [Symphoricarpos racemosus Michx., var. luevigatus Fernald.] Oceasionally established in fields and roadsides.

Lixpied borentis L., var. axericaxa (Forbes) Rehder. Common in damp mossy woods.

Vibutadar aliffolitar Marsh. Frequent in moist woods.

Trberacar acerifoliter T. Rich moist woods, Gray's Core, Reach, Deer Isle (No. 2773).

Vibericar dextatiar I. Rare. Wet banks of streams in woods, Atlantic, Swans Island (No. 2395).

Viburauar cassinomes L. Very common in bogs, swampy woods and thickets.

Sambecus caradexsis L. Frequent in wet meadows and thickets.

Sinnecrs racexosa L. Common in dry fields, clearings and open woods.

\section{CLCTRBITACEIE}

Enimocistis momita (Michx.) T. \& G. Infrequent. Escaped from cultivation, Naskeag Point, Brooklin (No. $1362)$.

\section{CANPANULACEAE}

Gapayela raputortoides L. Occasional. Escaped from cultivation. Roadsides, Naskeag Point, Brooklin (No. $992)$; fields, South Deer Isle (No. 914).

Chapaxtla rotudifolia L. Rare. Rich woods and dry open ficlds near serpentine quarry, Reach, Deer Isle (Nos. $19+4,21 \pm 7)$.

1. Rhodora 16: 119 (1914). 


\section{LOBELIACEAE}

Lobelia spicata Lam. Rare. Swampy meadow, Atlantic, Swans Island (No. 2247).

Lobelia inflata L. Common along roadsides and in fields.

Lobelia Doptanaxia T. Frequent on sandy and nunddy pond shores.

\section{COMTPOSITAE}

Eupatoriuar purpereum L., var. alaculatear (L.) Darl. Rare. Wet swampy woods, South Deer Isle (No. 18:3t). Eupatorium perfoliatum L. Common in wet meadows and thickets.

Solidago bicolor L. Abundant in dry sterile soil.

*Solmago Raxdir (Porter) Britton. Isle an Hant (J. C. Arthur).

Solidaso Prbertlas Nutt. Frequent in open fields and clearings.

Solddigo uligixosa Nutt. Isle an Haut (J. C. Arthur).

Solidaro sempervinexs L. Abundant on headlands, rocky shores and heaches and in salt marshes. A white-raved form has heen found at Isle au Haut by N. T. Kidder. ${ }^{3}$

Solidago Juncea lit. Frequent in dry open woods and fields.

Solidago neglecta T. \& G. Rare. Fields and pastures, Naskeag Point, Brooklin (No. 1329).

Solidago uxiligulata (DC.) Porter. Common in sphagnum hogs.

Solmago rugosa Mill. Abundant in fields and thickets.

1. Rhodora 19: 20 (1917). 
var. rillosi (Pursh) Fernald. Common in moist thickets and open woods.

Solidago xemoralis Ait. Abundant in dry sterile soil.

Solidago CANADExsis L. Common in damp thickets and open woods.

Solidago serotina dit. Rare. Roadside thickets near Torrer Pond, Deer Isle (No. 3097).

rar. Gindxted (Ait.) Gray. Moist alder thieket, Gray's

Cove, Deer Isle (No. 1907).

Solmago gramixifolia (L.) Salisb., var. Nutralum (Greene) Fernald. Very common. Dry fields and roadsides.

Aster macrophyluts L. Common in dry open woods and elearings.

Aster raptla L. Common in bogs and wet woods.

var. strictes (Pursh) Gray. Oecasional in bogs and exposed situations along the shore.

*Aster cormifolits I. Infrequent. Wooded banks, Isle au Haut (J. C. Arthur).

Aster laterifloris (L.) Britton. Tery eommon in dry fields, elearings and thickets. A very variable speeies often closely approaching var. hirsuticaulis (Lindl.) Porter. Isle an Hant ( $\mathrm{N}$. T. Kidder).

Aster Paxiculates Lam., var. Bellidiflores (Villd.) Burgess. Rare. Open places in dry woods near Head Harbor, Isle au Haut (No. 2410).

* Aster lovgifolies Lam. Isle an Mant (J. C. Arthur. N. T. Kidder).

Aster xovi-betgrl L. Very abundant in wet meadows. nswally near the shore. Common on headlands, rocky shores and borders of salt marshes. 
Aster puxiceus L. Rare. Marshy ground by roadsides, Naskeag Point, Brooklin (No. 24t2).

Aster vareluates Mill. Common in moist thickets.

Aster ncrimatus Michx. Frequent in moist woods and elearings.

Aster xemoralis dit. Frequent in sphagnmm bogs.

var. Blaker Porter. Loeally common in moist woods, Atlantic, Swans Island (Nos. 2970, 2930); near Lovers Lane, Isle an Hant (No. 304.3).

Erigerox ramosts (Walt.) BSP. Oeeasional in fields.

var. septextrioxalis Fernald \& Wiegand. ${ }^{1}$ Common in fields and pastures and roadside thickets.

Erigerox caxadexsis L. Infrequent. Gravelly bluff along shore, Irish Point, Swans Island (No. 2720) : sumny hillside, Little Deer Isle (No. 2581): Ocenurille, Deer Isle (A. H. Norton); Isle an Hant (J. C. Arthmr).

Axtexparia Parmini Fermald. Rare. Dry fields at edge of woods, Gray's Cove, Deer' Isle (No. 2079).

Axtemparia caxadensis Greene. Common in dry fields and open places.

Axtexparia Brannerdil Femali. Rare. Open woods, slopes of Sawyer's Momntain, Isle an Hant (No. 1698).

Axtexparia neonofo Greene. Common in fields and dry open woods.

Axtexparia xeglecta Greene. Rare. Fields and pastmres, Naskeag Point, Brooklin (No. 1421).

Axtexyaria petaloidea Fernald. Ocensional. Dry roadsides, Reach Road, Deer Isle (No. 217 7 ).

1. Rhodora 15: 60 (1913). 
rar. subcorrarbosa Fernald. ${ }^{1}$ Rare. Dry fields and roadsides, Fish Creek, Reach, Deer Isle (No. 2207). This variety is known in Maine only from Mt. Desert, Milford and this third station at Deer Isle.

Axapimats margaritacea (L.) B. \& H. Common in dry fields and clearings.

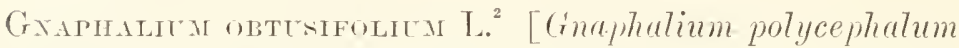
Michx.] Occasional in fields near shore and on headlands.

Graphaliux thigrosuar L. Abundant along roadsides and in cultivated ground.

Ira xaxtiffolia Nutt. Rare. Dry fields, South Deer Isle (No. 1St1).

Axbrosia artemismfolia L. Common in dry soil. Roadsides and enltivated ground.

Rumbeкia mirta L. Infrequent. Fields and pastures.

Bunexs froxmosi L. Common in muddy roadside ditehes and low ground.

Bidexs cervia L. Frequent in wet places.

Achilfea Millefoliea L. Very common in dry soil. A form with rose colored petals is quite frequent.

Achilfea laxtlosa Nutt. Rare. Open meadow, near shore, Seal Core, Swans Island Head, Swans Island (No. $677)$.

Axthenis Cotcla L. Common along roadsides and in waste ground.

Matricatia inodora L. Roadsides. Isle au Haut (No. $3064)$.

1. Rhodora 16: 133 (1914).

2. Sp. Pl. 2: \$51 (1753). See Blake, S. F., Rhodora 20:71 (1918). 
Matricarla soaveolexs (Pursh) Buchenan. Very common about dwellings and along roadsides.

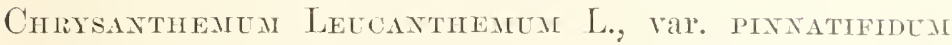
Lecoq \& Lamotte. Tery common in fields and along roadsides.

Taxacetar vegare L., var. chispuar DC. Infrequent. Fields and roadsides. Escaped from cultivation.

Artemisia velanis L. Occasional in fields and waste places.

Emecitites meracheola (L.) Rat. Infrequent. Gravelly soil of upper beach. Gray's Core, leer Isle (No. 1935).

var. intermeds Fermald. ${ }^{1}$ Common in moist woods and clearings.

Sexecio syluatices L. Common on gravelly beaches.

Sexecio Robbixsi Oakes. Rare. Swampy meadow, Reach, Deer Isie (No. 21:36).

Aroticar xearonosuar Lejeme. ${ }^{2}$ Rare. Roadsides near Long Pond, Isle an Fant (No. 1240), also ( X. T. Kidder).

Aroticu maxes Beruh. Common around dwellings and on waste ground.

Cinsiem laxceolatex (L.) Hill. Common in fields and pastures.

Cinsma antioum Michix. Firequent in moist woods.

Cimsical arvexse (L.) scop. Tery common. Fields, roadsides and cultivated wround.

Cextacrea Jacea L. Rare. Fields and pastures. Taskeag Point, Brooklin (No. 1023).

Cichorium Ixtracs L. Rare. Wiste ground near sardine factory, Center Harbor, Brooklin.

1. Rhodora 19: 27 (1917).

2. Lejeune \& Courtois, Comp. Fl. Belg. 3: 129 (1836). See Femald, M. L., and Wiegand, K. M., Rhodora 12: 45 (1910). 
Leontonon altumalis L. Tery common in fields, waste ground and along roadsides. A form with greenishyellow heads without lignles occurs in cultivated ground near Gray's Cove, Deer Isle (No. 2533).

Taraxacum officianle Weber. Frequent. Roadsides and fields.

*ar. palitstre (Sm.) Blytt. Roadsides, Barter's Store, Isle an Haut (J. C. Arthur).

Taraxacuar eriturosperaura Andrz. Common. Fields, pastures and roadsides.

Soxcints arvexsis L. Frequent on gravelly seashores.

Sonchus oleracers T. Common in waste ground.

Soxchis asper (L.) Hill. Occasional in waste ground, often near the shore.

Lactuca canafexsis I. Occasional. Mroist woods, Atlantic, Swans Island (No. 2387) ; clearings, Reach, Deer Isle (No. 30.38) ; dry roarlsides near Moore's Harbor, Isle au Haut (No. 1691).

Lactuca minsuta Mruhl, Infrequent. Clearings, North Brooklin (No. 1750); open hillside, Little Deer Isle (No. 2.585).

Lactuca spicata (Lam.) Hitche. Common on low ground. Prenanthes trifoliolata (Cass.) Femald. Abundant in dry open woods, clearings and fields.

Prexixties altissina L. Rare. Rich rocky woods, Isle an Haut (No. 126t); moist rich woods, Gray's Cove, Reach, Deer Isle (No. 2862).

var. inspmula Fernald. Infrequent. Rich moist woods, Hockomock Head, Swans Island (No. 266r); Gray's Cove, Reach, Deer Isle (No. 2865); Lovers Lane, Isle an Haut (No. 3046). 
Hieracium alraxthatal l. Common in fields and pastures.

Hierachar pratexse Tausch. Tery common. Fields and roadsides.

Hiemacilas scabreas Michx. Frequent in dry woods and clearings.

Heraciua caxaderse Michx. Common in thickets at edge of woods and in clearings and fields.

\section{SuMmary of the Flora}

Species Varieties Forms Total

Pteridophytes

Gyanosperiss

Moxocotylenons

Dicotrledons

Archichlahimeate

Metachilanipeae

Native Plaxts

Ixtrodecen Plaxts
$3: 3$

12

160

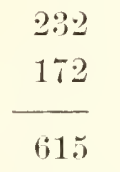

Species

$$
495
$$

120
6

1

46

.

13

39

217

\begin{tabular}{rrrr}
34 & 1 & 267 \\
33 & 6 & 211 \\
\hline 120 & & - & \\
\cline { 3 - 3 } & 12 &
\end{tabular}

Varieties Forms Total

$\begin{array}{rrr}107 & 10 & 612 \\ 13 & 2 & 135\end{array}$

PHYTOGEOGRAPHICAL ASPECTS OF THE FLORA TIE DISTRIBUTION OF THE NATIVE FLORA

An examination of the ranges of the 612 native plants of the eastern Penobscot Bay region shows that the flora falls more or less readily into six groups:

Group I. Widespreat Plaxts, occurring thronghout eastern North America. 
Groep II. Boreal Plaxts, species which extend to Labrador, Greenland and the Far North.

Group III. Caxamin-Alegghaxiax Plaxts, which extend northward only to eastern Quebec or Newfoundland and represent a transitional group between the horeal species and the more sonthern forms.

Grodp IT. Allegindix Plaxts, sonthem species which reach their northern limit in eastern Maine or adjacent Tew Brunswick and Nora Scotia.

Grorp T. Carolixiax Plaxts, which represent a northward extension of the Atlantic Coastal Plain flora.

Groep VI. Local Plaxts, which are either species with a limited range or isolated varieties of more widely distributed species.

This gromping of the Penobseot Bay flora is a modification of the classification of life zones of North America, proposed by Hr. ('. Hart MLerriam. ${ }^{1}$ In that part of eastern North America with which we are dealing Nerriam recognizes the following regions and life zones:

Bolient Reriox: comprising the northward part of the continent with a narrow sonthward extension on the higher mountains.

1. Arctic-alpine ane-above the limit of tree growth.

2. Iludsonian zone-the northern part of the coniferous forest region which extends from Labrador to Alaska and south in a strip on the higher mountains.

3. Canadian zone-the southern part of this forest region, which comprises Canada, northern New England and New York and a greater part of the mountains.

1. Merriam. Life Zones and Crop Zones of the United States, U. S. Dept. Agr. Biol. Survey. Bull. 10. 1898. 
Arstral Regrox: comprising practically all of the United States save the boreal mountains and the tropical lowlands.

4. Transition zone-where the horeal and austral resions overlap, with southern species at their northern limit and boreal species at their southern limit. In the East this zone is represented by the 17leghanimen area which extends from New England and southern Ontario west to the Dakotas and somth along the Alleghanies to Georgia.

$\therefore$ Cpper austrul zone-consisting in the east of a humid area-Carolinian-which oeeupies a laree part of the Middle States at low altitudes, and on the Atlantic coast extends from Chesapeake Bay to southern New Enwland with arms up the river vallers.

In the eastern Penobseot Fay region, where these zones orerlap, it is impossible in many cases to distinguish clearly between species which are ('anadian and those which show an Alleghanian trend. It is equally hard to draw a definite line between the varions sub-ilivisions of the Boreal Region as interpreted by Merriam. Consequently it has been thonglit best in the present discussion to consider the more northern of the boreal plants, i. e., the Aretic-alpine and Hudsonian, as distinet from the (anadian, and to place them in a group by themselves (II). The Canadian plants are mited with the transitional Alleghanian forms in a seond group (III). Some of the Alleghanian plants, howrere, are perfectly distinet from these northern species and are considered separately in Group $I V$. Inother group $(V)$ is composed of sonthern species which are clearly of Carolinian origin.

To summarize then, the native flora of the eastern Penohseot Bay region will be considered nnder the following hearls: 
I. Widespread Plants-5t species, a per cent of the flora.

II. Boreal Plants-95 species, 16 per cent of the flora.

III. Canadian-Alleghanian Plants-353 species, 62 per cent of the flora.

TV. Mlleghanian Plants-56 species, 9 per cent of the flora.

V. Carolinian Plants-18 species, 3 per cent of the flora.

VI. Local Plants-6 species, 1 per cent of the flora.

The relative importance of the various groups in the makeup of the flora is shown graphically in Fignre 1. As wonld le expected from the location of the eastem Penobscot Bay region the largest pereentage of its flora consists of CanadianMleghanian species.

\section{Groep I. Midesprean Plaxts}

It will be possible to dismiss this group with but little comment. Its it species are rery widely distributed and occur in varions habitats, for the most part throughont eastern North Imerica save in the extreme north. As examples may be cited :

Polypodium vulgare

Equisetum arvense

Typha Tatifolia

Agrostis hyemalis

Carex scoparia

Jumens bufomins

Polygommm sagfitatum
Cardamine pemsylranica

Callitriche palustris

Teronica serpyllifotia

Orobanche uniflora

Plantago major

Erigeron canadensis

lathen Millefolium

\section{Gromp II. Borenl Plaxts}

This group comprises 95 plants of the Aretic-alpine and Hudsonian zones according to Merriam's classification. In phrtogeon aphical literature the terms aretic, sub-aretic, arctic-alpine, alpine and suli-alpine have all been used more 
or less indiseriminately to apply to the flora of the norther'u regions. This multiplieity of terms is a result of the dificulty experieneed in attempting to place these boreal plants,



Figulis 1. - iraph showing the relative importance of the various elements in the eastern Penobscot Bay tlora.

especially those which extend to the Aretie C'ircle or filrther north, in any arbitrary category. Since very few of these species have the same distribution, anthor's have been forced 
to employ different terms in attempting to classify them satisfartorily, and this has led to considerable confusion. In the present discussion the term sulh-aretic will be employed in referring to such of these plants as occur in the Penobscot Bay region. for no strictly aretic forms are ineluded among them. A more sonthern element of the boreal flora, on the other hand, is much more definitely marked off and the term Hudsonian is miversally nsed to refer to those species which range to the Labrador Peninsula and IIndson Bay and attain their maximm development in eastern C'anada, but which fail to reach the far north.

The bnreal flora as a rule is widespread in northern regions, lut gradually becomes restrieted toward the sonthern limits of its range, where it neenrs only Joeally in the most favoralle situations, such as high momtain tops, sea e]iffs or cold bogs. The eastern Penohseot Bay region ocenpies a midway position, where the horeal plants are still fairly widely distributed, hut even here certain of the more northern species are uniform] restricted to the coastal eliffs and leadlands or to hogs.

\section{Sib-irotic Plaxts}

The most interesting frature from a phytogengraphical pnint of riew in the Pemobset Bay region is the ocemrence of several species of sub-aretic plants on the cold hearlands and sea-cliffs of the onter islands. The extreme exposure of these shores, the constant drenching with cold spray to which they are subjected, and the frequent presence of fogs all eomline to render ennditions quite like those in aretic regions and funish a habitat where the horeal species can exist.

The presence of this flora on the eastern Maine enast and in adjarent New Brunswick has long been recognizerl. As early as 1869 r. F. Watther: pullished an acenut of the 
occurrence of aretic plants in New Brunswick. In 1894 Rand and Redfield emphasized the presence of boreal specics on Mrount Desert and the C'auberry Isles ${ }^{2}$, and more recently Dr. George G. Kemnedy noted their existence at Cutler. ${ }^{3}$ Careful observations on the part of other botanists have resulted in a considerable increase in the number of species and an extension of their ranges toward the south.

In 1910 Fernald and Wiegand", writing of enditions in Washington County, Maine, stated that "in general this narrow coastal sub-aretie strip extends along the onter mainland and islands to Great Cranberry Isle, and in less pronounced derelopment to Tonhegan and even heyond."

The reason for this less pronouneed development is readily apparent. Eastward from Mount Desert the shores of the mainland itself are bold and have a full exposure to the eold sea. while the ontlying islands are comparatively near at hand. Consequently it is possible for these boreal plants to have a fairly contimuons distribution along this part of the coast. Test of Mount Desert, however, the mainland is many miles from the open oeean, owing to the deep indentation made by Penobseot Bay. The proper halintat for these species in this region is found only on the fringe of onter islands which are extremely isolated from the mainland and from one another. As a result, many species, such as Scirpus caespitosus. Montia lamprospermu and Rubus Chamaemorus, which extend to Mount Desert or the neigh-

1. Matthew. On the Occurrence of Arctic and Western Plants in Continental Acadia. Proc. Nat. Hist. Soc. New Brunswick. April 13, 1869.

2. Rand \& Redfield. Flora of Mount Desert Island, Maine, p. 21. Cambridge. 1894.

3. Kennedy. The Maine Coast at Cutler. Rhodora 4: 23-26 (1902).

4. Fernald \& Wiegand. A Summer's Botanizing in Eastern Maine and Wesern New Brunswick. Rhodora 12: 101-121, 133-146 (1910). 
boring seaward islands are malhe to cross the interreninge

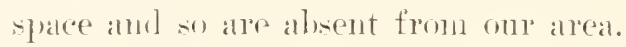

1 considerable number of sub-aretic species, nevertleless. are to be fomnd on the onter headlands of the Penolseot Bay istands. Crowing in humus or peaty turf are such plants as: Empetrum nigrum, of ten projecting ont orer the exposed rocks in great mats: Funiperus horizontalis, frequent on the onter islands and also known fron two stations at Brooklin, some ten miles up the Bely : and Tareinium Titis-Then, var. mims. (onnmon on barren hills and onterops as well. Other species are num less frequent: Serfum rosem. erowing in great profusion on clifts at Placentia Lstand near Swans Tsland, and on a rocky beacte on Isle an Tant: Tris setosa. vatr. remedensis, on the crests of headlands at Black Point, swans loland, a sontluard extension of range fron little Thek Istand ; ${ }^{1}$ and A renaria groonlandicn, at a singte station each on leer lsle and Isle an Hant.

The raised bugs bark of the headlands, a characteristic feature of this coastal strip to the eastward. are larking in Penobsot Bay or at most attain only a meagre development and so are malle to support the plants common to such a habitat. It is perhaps a significant fact that most of the sulb-aretic plants which fail to reall onr alrea are species of these raised bogs or heaths, which would he mable to find the neersing condition for their existence eren if they should bridge the gap betwean Momnt Desert and the P'enohscot Bay islands.

The rocky heaches and hackish marshes atso furnish a decidedly boreal habitat fur surh plants as C'are.r moritima,

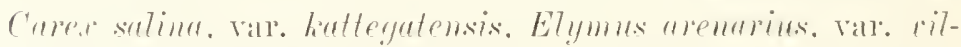
losas. Coeloplentrum actapifolium and Mertensin maritione.

1. See Norton, A. H., Rhodora 15: 13 (1913).

2 . See Fernald ot Wiegand, Rhodora 12: 106. 
The development of this sub-arotic Hora is much less pronomnced west of Pemobseot biy and the ferr spocies which

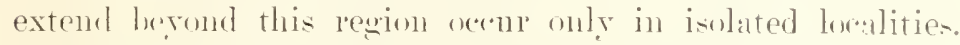
Is examples may he cited: , sedum rosenm, ratchine only to Monhexan: C'arer sulima, vald. Rattegatensis. to the Buston Basin: Carex maritima. to Marshtield. Mass.: and Elymus aremerins, var. villosus, to P'rorincetorn. Mass.

The trpical ramge of these sub-aletic species, which ale widespread in the far North lut restriotod tomald their sonthern linits, is well illustrated by Empetrum nigmom (Fig. 2).

\section{HIMUONIAN P'LANT'}

The Hudsonian elemont of the horcal Hora in the eastern Penobscot Bay region ofters little of phytogeographical interest. The speries are comparatively wides]read thromghont the area, ocemring in many different hatitats and forming a conspicuons part of the vegetation. The plants of this zone frepuently grow in company with sultraretic trpes in sold habitats as sea rlifts and sphasum hogs. Fren more often they are found interningled with Camalian or dlleghanian species and might he classified with them were it not for the faret that the Indsonian fioral ranges farther north and so is considered distinct as regards distribution.

In order to wive a wencral impression of the Hudsonian elenent in the flora of the eastern Penobscot bay region it will he sutticient to mention merely a few of the species

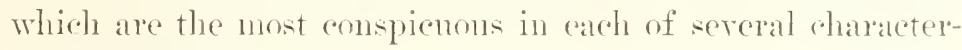
istic halitats.

\section{I'LANB) FURESTS}

Mryopteris spimulosa

Lyropodium amotimum

Equisetum syliationm
Pyrns ameriramen

liubns jubescens.

Cormus comatensis: 
Picea canadensis

1bies balsamea (see Fig. 3) Clintonia borealis

\section{Coptis trifolia}

libes prostratum
Moneses uniflora

Trientalis borealis

Gratium triftorum

Limnaea boreatis,

var. americana

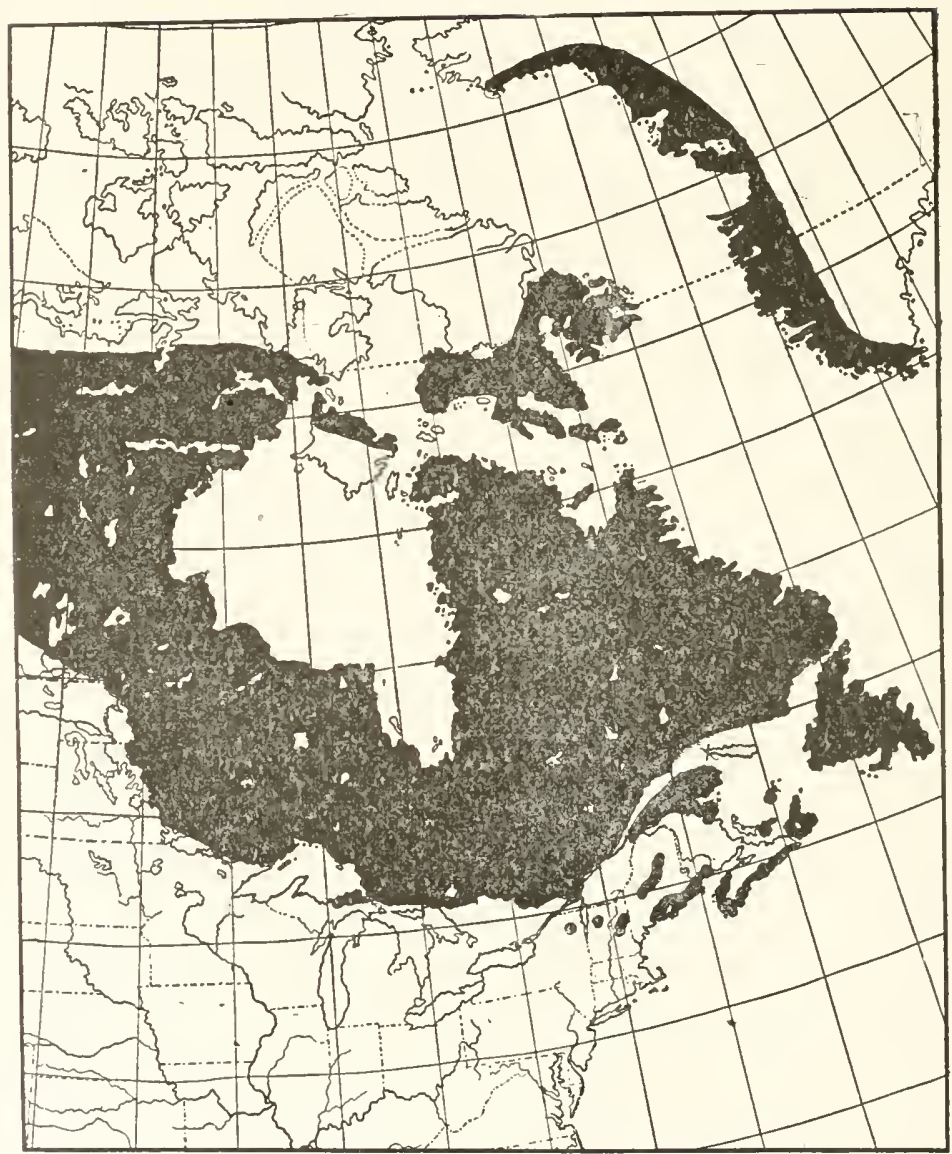

FugrRe 2.-Distribution of Empetrum nigrm, a representative aretic-alpine species. 




Fiare: :-Distribution of Abies balsamea, a representative Hudsonion species.

OPEN HEADLANDS

Deschampsia flexuosa Festuca rubra

Spiranthes Romanzoffiena

Larix laricina

Picea mariana

\section{PEAT BOGS}

Cerastium arense

A renaria lateriflora

Potentilla tridentata

Ledum groenlandicum

Lalmia polifolia 
Eriophorum cullitrir

C'arest panpercula,

var. inrigue

Smilarina trifolia ('hamaedaphne calyoulata

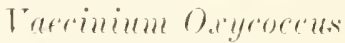

Loniceru crusulen,

rar. collesesens

STIAIPS

C'ared rootenowii

Epitobium palustre

Myirn linle

Menyanthes trifolintu

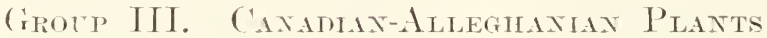

Is wonld be expected from the location of the eastern Penobscot Bay rewion, the greater part of its flora consists of Canadian-Alleghanian speeies. The 38:3 plants which belong to this transitional gromp comprise ti2 per cent of the native flora and eontribute the most conspicuns features to the regetation of the region. The two elemonts which go to make up this group are by no means distinet. In faet in most instances it is impossible to say with perfect aecuraey whether a species is Canadian or Mleghanian sinee its distribution does not coineide with either of the arlitrary zones of Merriam. For the purpose of the present disension, however, it is not necessary to draw hard and fast lines betwren these two elements. The signiticant point for us is that the speeies of this gromp extend northward to eastern ('anada, northern New Brmuswick and Tora Scotia, or even Newfomdland.

Althongh originally applied to the so-ealled life-zones, the terms Camadian and Alleghanian are in more frequent use at the preant time in referring to two of the well marked forest areas of the Tnited States. Is ('ooper emphasizes. eastern North Ameriea is divided into two great phytogeographical areas - the easteru leciduous forest and the north-

1. Cooper. The Climax Forest of Isle Royale, Lake Superior, and its Development. Bot. Gazette 55: 1-44, 115 140, 1S9-235 (1913). 
eastern "oniferons forest. The former is charaleteristic of the Alleghanian region, the latter of the Canadian region. By far the areater part of the forests of the eastern l'enobseot Bay region belong to the lattes type, which attains its maximmm development in eastern (anada.

\section{Canablat Elemext}

Mthongh two of the dominant trees of the Canadian forest, Jiren commensis and dbies bulsemen. together with sereral of the associated species, are nore strictly. Hudsonian in nature, since they extend north into Labrador, there are a much later number of characteristic species which answer the requirenents for canalian distribution. Anomg these may be mentioned the following trees and shruls:

Taxus comadensis Populus tremuloides

Picea rubru Ilex vertirillatu Pimus resinosen Acer spicatume Betulu ulbu, Cormes alternifoliu var. papyrifera Diervillu Lonier ru 17uns incenue riburmum ressinoides

Herbaceons forms characteristie of the Canadian Forest include:

Mryopteris cristatn

Lyeoportium lucidulum

streptopus roseus

libes hirtellum

\author{
Datiburda repens \\ Oxulis americuma (see Fig. t) \\ Tiola incorguita \\ I ralin undicuntis
}

The Canadian plants are not restricted to the wondlands, however, but oecm in every possible habitat. Only a few of the nore common species can be mentioned in this remnection. 


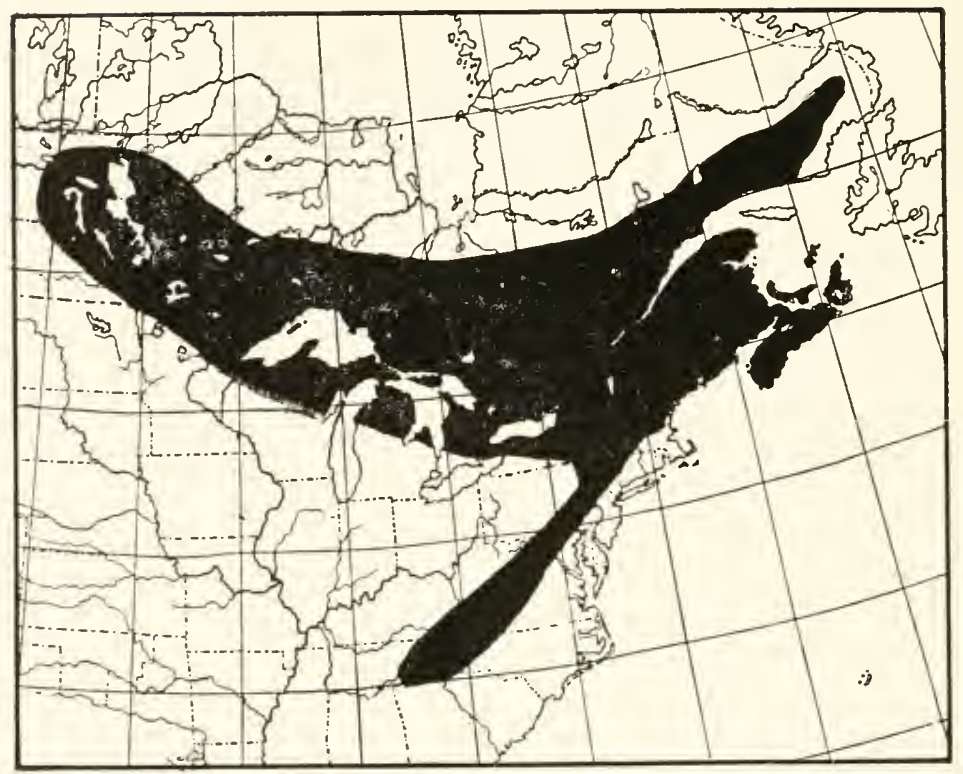

Figl'RE 4.-Distribution of Oxalis Americana, a representative Canadian species.

FIELDS ANJ CLEARINGS

Panicum boreale

Carex pallescens

Polygonum cilinode

Prunus pennsylianica.

Oenothera pumila

Aralia hispida.
Taccinium pennsyleanicum

Euphrasia americana

Rhinanthus Crista-galli

Sambucus racemosa.

Prenanthes trifoliolata

Hieracium canadense

SWAMPS

Temopanthus mueronata

Impations biflora

Tiole cucullata

Lysimachia terrestris 
Thalictrum polygamum

Lathyrus palustris, var. pilosus scutellaria galericulata.

Galium trifidum

Mentha arvensis

\section{Alemghaniax Elemext}

The second of these phytogeographical regions - the eastern deeiduons or Alleghanian forest-has its center of distribution in the Ohio Valley and the southern Appalachians. This Alleghanian zone proper does not extend far into New England, but under favorable envirommental eonditions eertain of its speeies have been able to exeed the general limits and advance northward to eastern Maine and the Maritime Provinees, and in some eases even to Newfoundland. Only those speeies whieh extend to Cape Breton or Newfoundland are included in this group. Typieal examples include:

Pinus Strobus

Smilacina racemosa.

Medeola virginiana

Trillium undulatum
Betula lutea

Fagus grandifolia

Acer rubrum

Prums virginiana.

A detailed diseussion of this element of the CanadianAlleghanian flora will be taken up in eonnection with the more strictly Alleghanian plants in Group IV.

\section{Grovp IV. Alleghanian Plaxts}

In eontrast to the species of the two previous groups, which represent for the most part a boreal flora approaching its sonthern limits, there exists in the eastern Penobseot Bay region another group which represents an austral flora at the northern limits of its range. This group of 56 species, 9 per cent of the flora, consists of plants which have a wide range to the sonthwest but northward reach only to eastern New England and adjacent Canada. 
1. has been stated, the Alleghanian zone proper does not extend far into New England, but the influence of the ocean renders conditions along the coast equable enongh so that some of theme anstral forms have exceeded their general limits and pressed much farther northward. The distribntim of these outpost species is by un means contimuns and the plants ocemr in isolated localities, a heharior characteristir of species at the edge of their monge. Is we have noted. rertain of these sonthorn forms are to be formd in cape Breton and Newfomdland. These are inchnded in the preceding eroup since it is hard to distinguish them from Canadian species, and mly those forms which attain their unrthern limit in Maine, New Prunswick or sonthern Tora Scotia and which show a definite Alleghanian distribution are considered as belonging here.

The habitats of these Alleghanian species in the eastem Penolseot Pay region vary. Some of them oecor in wet places: Tascinium atrocorcum, Fraximus penusylmanien. and Tiburmum dentutum in swampy woods: Derorton repticit-

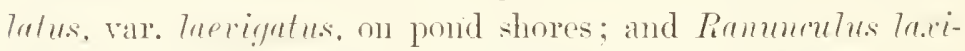
rnulis. locally common in muddy bog holes at South Deer Isle, its present northern limit.

Other species frepment dry open tields: Lilim phitartetthicum. Polygula sanguinen, and P. resticillalu. Lechen intermediu, Lysimachien yuadrifolin, and Antemurin Parlinii. still others are found in the dry leaf monld of wonds: Oaliesin sessilifolin, Polygomutum biflorum, and Pedienluris renudensis.

The striking featmre in comnection with the Alleghanian Hora is not the presence of these few isolated plants of swamps and dre woods but rather the almost eomplete absence of the

1. Griggs. Observations on the Behavior of Some Species at the Edges of their Ranges. Bull. Torr. Bot. Club 41: 25-49 (1914). 
more characteristic spccies of the rich wonds--the eastern deciduons forest. In stch a forest the dominant trees are Aer sucrharmen, Fingus armurtifotin. Betula Tutea, Tsuga conurtensis. and P'inus strobus, and associated with them

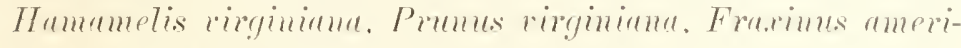
rana, with feer rubrum in swampr woods.

Althongh these species oecur northward and even attain considerable development in favorable howaties, they are very infrequent in the eastern Penohiont Bay region, an area characterized by granites, schists and other rocks which, owing to their high content of silica, vield a sterile or acid rather than a basic soil.

Eren more noticcable is the absence of the herbacens types of the rich wonds, the spring flowers common in leaf monld. Among such forms which are entirely mising from the eastern Pemolsent Bay region may he mentioned

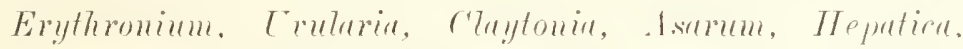

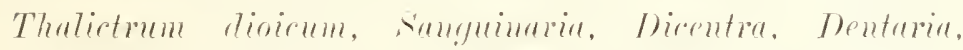
C'aulophyllum, Vitella and Penuer. These plants and many others extend thromgh New England as far as the Kennebec River, leave the coast at that point and follow up the limy valley of the Kemmehee to the rich Silnrian linnestone area of northern Mane and eastern Canada. Tibumum dentatum illnstrates the distribution of the Alleghanian speries, which extend northward only to New Brunswirk (Fig. i).

The absence of these plants of the Alleghanian forest from the eastern Penobsot Bay region has two noteworthy exceptions. In the northern part of Deer Isle there exists an area of serpentine and another of greenstone, a basic igneons rook. In these areas the repetation is entirely different from that of the surronnding anuntry and consists of decidnous trees and their characteristic nndererowtls. Betuln huten, Furyus smoutifotin and iser rubrum predoninate and in the deep 


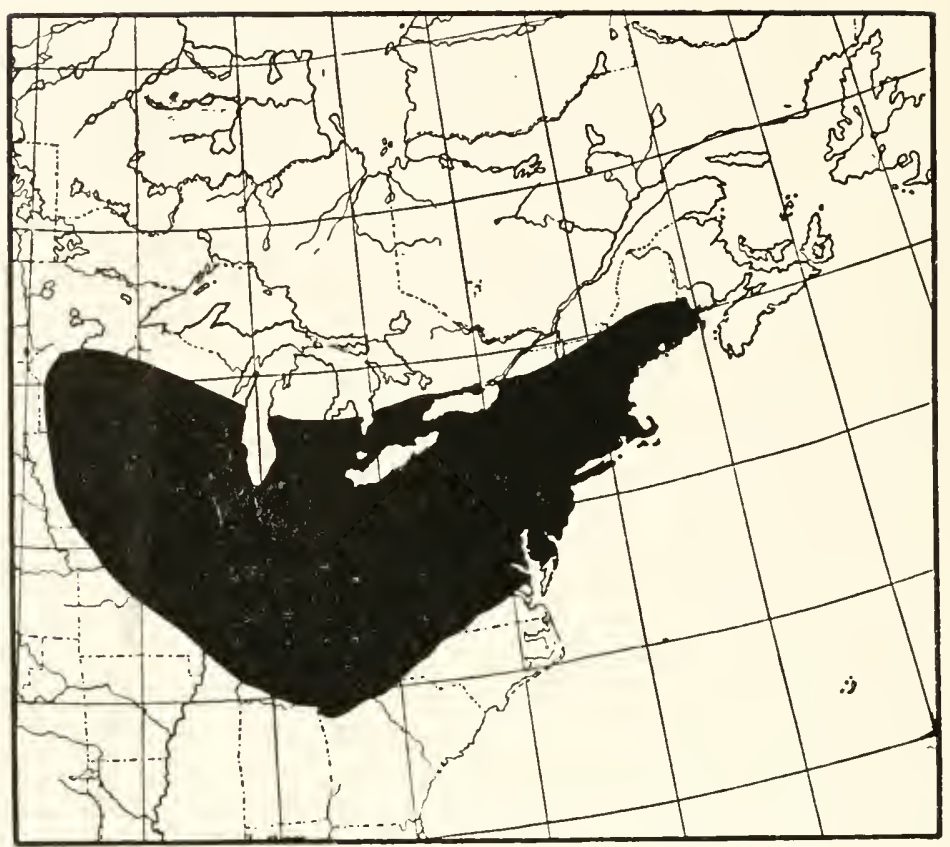

FIGIRE 5.-Distribution of Tiburnum dentatum, a representative Alleghanian species.

leaf mould beneath these trees occur Adiantum pedatum, Actuea rubru, Carex novae-angliae, and other speeies found nowhere else in the region. The reason for the restriction of the species to these areas is readily apparent. They are plants which require a good percentage of basic material and they are mable to olotain the necessary food in the acid or neutral silicions soils which prevail elsewhere. As will be shown later, these plants should be considered as relic rather than pioneer types, and they persist here only because of the extremely farorable envirommental conditions. Other Alleghanian species are more tolerant than these rich woods 
forms and can mantain themselves, and even advance, in areas with a much smaller percentage of hasic material. Even these plants, however, aroid the loose soil of the dry coniferons forests and the arid hogs.

The occurrence of the above mentioned species on a serpentine area brings us to another problem. Tsually a serpentine region is characterized by a sreat pancity of regetation and the presence of peculiar species which are ahle to ntilize, as food, the magnesimm, an element which is extremely toxic to most plants. It first sight it wonld appear that here was a condition directly opposed to the general r'tule. Far from leing a sterile area almost devoid of regetation, this area has the richest floral of any part of the region. It is apparent that some other sulstance nust be present in the soil to comnteract the effect of the magnesimm. In examination of the mulerlyine serpentine rock shows it to be filled with numerons reins of calcite which readily decomposes and furnishes lime to the soil. The work of Von Rammer and of Loew has demonstrated that ralcinm experts an antagnistic action on magnesimm and may eren destroy its toxicity entirely. In this comnertion Osterhent" states that "a single Ca ion acts as an antidote to from 20 to 100 ions of Ta.K.XH, ete" The lare amount of calcite present in this serpentine wonld easily he sufficient, not only to connteract the magnesimm, but alon to render the soil extremely basic and fertile.

1. Von Raumer. Calcium und Magnesium in der Pflanze. Landw. Versuchsst. 19: 253-280 (1883).

2. Loew \& May. The Relation of Lime and Magnesia to Plant Growth. T. S. Dept. Agr. Bur. Plant Ind. Bull. 1 (1901); Loew. The Plipsiological Rôle of Mineral Nutrients in Plants. T. S. Dept. Agr. Bur. Plant Ind. Bull. 45 (1903).

3. Osterhout. Some Chemical Relations of Plant and Soil. Science N. S. $36: 571.576(1912)$. 


\section{Gigorp T. Caroltalax Plaxts}

This small sromp of southern species-:; per cent of the native flora of the eastern Penobsent Bay region-affords nearly as much phrtogengraphical interest as do the subaretic types. These Camolinian plants, althongh of sonthern origin, are distinct from the Alleghanian. 'Ther are characteristic of the Cretaceons and Tertiary sands and clays of the New . Tersey Pine Barrens and other portions of the Atlantic coastal plain. This coastal plain flora extends northmard from the Gulf of Texien along the coast to Xew Jersey and thence, in less prononneed derelopment, to staten Island. soutlern Long Tsland and sontheastern New England. with arms rumning wy the lareer river vallers.

The presene of typical coastal plain species oroming on aranite rocks or the scantiest of soil on the Maline comst affords intresting speculation as to their geographic origin, the natme of which will he discussed later. The orourrence of Carolinian plants in such habitats, howerer. is not unipue, for sereral coastal plain plants have long leen known ou quartzite rocks in the Kittatimuy Mountains of New Jerser. These quantzite rocks and the aranite hills of Penobseot Ba! furnish a soil which. like that of the sand plains along the coast, is rery rich in silica and so is acid rather than basic. (sice palge 202. Sote 2.)

It lias been aroned that species which are restricted to these acid soils are able to ntilize the silica in some way as food and the term "silicophiles" has heen applied to then. Contejean," Hileward and others lave shown, howerer, that

1. Britton. On the Existence of a Peculiar Flora on the Kittatinny Mountains of Northwestern New Jersey. Bull. Torr. Bot: Club 11: 126-12S (1884). Note on the Flora of the Kittatinny Mountains. Ibid. 14:187-189 (1887).

2. Contejean. De l'Influence du Terrain sur la Vegetation. Ann. Sci. Nat. Bot. V. 20: 266-304 (1874); VI. 2: 222-307 (1875).

3. Hilgard. Soils, pp. 521-522. New York. 1911. 
these plants do not prefer the sterile soils, hut rather are repelled hy lime and other hasie clements and so an'e forced to seek refuge in a soil which is nentral or even acid.

Is has been stated, the majority of carolinian species rach their northern linits in the Tnited States in New Jersey or southeastern Tew England. Some, howerer, extend northward to the sandplains of southwestern Marine, and a few have presed on to the sterile soils and granite rocks of the eastern wast. Anomg suchl forms may be mentioned Pimus rigidu. Whird extends to Monnt Thesert, and Juncus Greenei. which reaches Rionpe Bhntfs in ITashington Comntr." Other speries. such as Copema Comratii and Hudsonia evicoirtes, attain their nopthem limit in the I'nited States in this region lunt reappear in Noval seotia and Newfommdland. I few other's have a continnons distribution along the const to Newfomulland, i. e., lster nemormlis (Fig. i).

Other ohatacteristic Camolinian speeses frepuently fomul in the eatstern Penolseot Bar region inelude:

\begin{tabular}{|c|c|}
\hline Potamoyeton Oakesimms & Eriocunton septaneputare \\
\hline Panicum impliratum & funcus effusus. \\
\hline Ammophila arenaria & solutus. \\
\hline C'are.t hormathodes & Myrien carotinensis \\
\hline
\end{tabular}

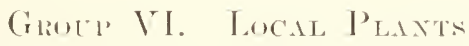

1 very few of the native plants of the region are extremely local in distribution. Crataegus . Fonesae is clearly endomic in this general locality, ranging only from Momut I lesert to Casco Bay, a distance of abont one hmuled miles. ()ther

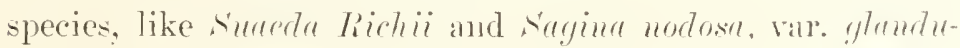
lose, extend only to Tora serotia on the one side or Marwat-

1. Knowlton, C. H. Rhodora 17: 154 (1915). 


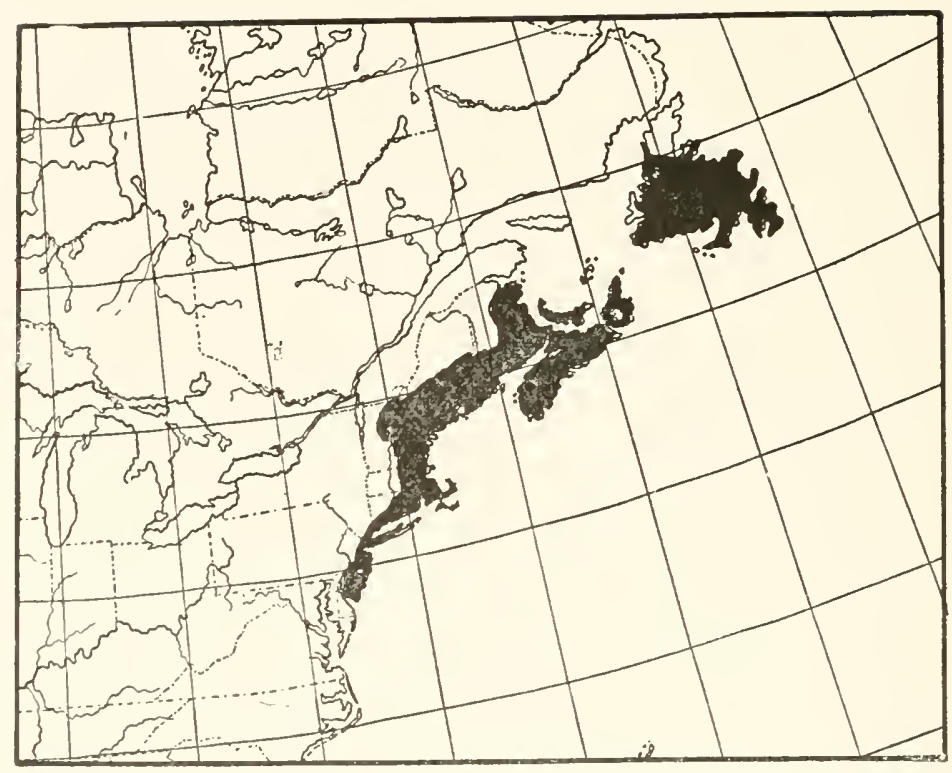

FriCRE 6.-I)istribution of 1 ster nemoralis, a representative Carolinian species, extending along the coast to Newfoundland.

chusetts on the other. Still others are isolated varieties of species more abundant in other parts of North America; -uch ats lombis Drummondii, var. comnexa, common on Crow Tublile at the eastem ent of Bradbury's Island near Deer Isle. With the exception of a station at Rivière du Loup, Qucluec. this is the only occurrence in the East of this plant of the momntains of western America. Another western plant, Comus rmutensis, var. intermertia, frequent on the sterile slopes of IIount Champlain. Isle au Haut. is known dewhere in the east only from Labrador and Newfoundland.

Thus far we have heen primarily concerned with the existing flora of the castem Penobscot Bay region. It will be well at this point to again call attention to sone of the more conspicnons absences among the native plants. 
The most noticcalle of these absences, that of the species characteristic of rich soils, has already been alhnded to. Such families as the Ranunculaceae, Cruciferae, Suxifugucene, Leguminosue and serophulariareae, to which many of our commonest speeies of the rich woods belong, are scarcely represented save by introduced species. The pancity of these Alleghanian plants in our region is brought ont more clearly by a comparison with a region where environmental conditions are more favorable. Kennedy's Flora of Willoughby, a limestone area in northern Vermont, lists 690 plants as against 736 in eastem Penobscot Bay. Of these 690 nearly 300, or to per cent, are lacking in our region, which is characterized for the most part by neutral and acid rather than basic soils.

I second noteworthy absence is that of species present on the mainland to the east and west and even on Mount Desert but which have failed to reach the islands of Penohsent Bay. As examples may be cited:

Agropyron canimum

Triglochin palustris

scirpus caespitosus

Carex norvegica.

Carex lenticularis

Juncus filiformis
Anemone quimprefolia

Chrysosplenium americamum

Polygala paucifotia

Ludvigia palustris

Chimaphita umbellata.

Lobelin rardinatis

These species it will be noted are not restricted to any one group, but include northern as well as sonthern forms. It is obvions from this fact, and from the eiremmstances of their occurrence in nearby localities on the mainland, where soil and climatic conditions are similar to those in Penobscot Bay, that some factor other than the character of the habitat

1. Kennedy. Flora of Willoughby, Vermont. Rhodora 6: 94-134 (1904). 
must be responsible for their absence from our area. A glance at the map shows the answer. In the Penohseot Bay region the coast is more broken up and the islands are farther remored from the mainland and from one another than anywhere else alony the Maine coast. This condition has been more pronouneed in the past than it is at present and it has been impossible for many plants which are present on the mainland to bridge the gap to the ontlying islands.

\section{THE ORIGIN OF THE NATINE FLORA}

Any discussion of the origin of the native flora of the astern Penobseot Bay region is natmally limited in seope, since all restiges of the pre-olacial flora were presumably destroved during the Iee Ige. Whatever the nature of this pre-existing flora, whether similar to that of the present time, or showing more sonthern tendencies owing to a wamer climate. it must have been driven ont of the region by the advent of the ice. A portion of this flora was probably. foreed into the sea, where it perished, but another part was driven south and had reached New Jerser and the sonthern Appalachians before the advance of the glaciers was ehecked.

Upon the retreat of the ice at the close of the Glacial Period these northern plants began to follow hack to their old homes from these centers of distribution along the $A$ ppalachians, for the most part "falling back into zones which long enviromment had impressed upon them.," Adans ${ }^{2}$ conceives of this northward migration' as necurring in wares. The first of these waves consisted of the Aretic-alpine specien which were able to live at the very edge of the ice-sheet in a

1. Bray. The Development of the Vegetation of New York State. N. Y. State Coll. For. Tech. Pub. 3 (1915).

2. Adams. Postglacial Origin and Migrations of the Life of the Northeastern United States. Jour. Geog. 1: 303-310, 352-357 (1902). 
habitat unfavorable to most plants. In their northward jounney two alternatives were open to these plants: either to follow the ice to the Far North, where many of them are widespread at the present time; or to remain behind in farorable situations, perhaps following the cold zone up the mountains or lingering in cold bogs or on exposed shores. The sub-arctic species in the castern Penobseot Bay region belong to this latter group of relic trpes, which were left behind in the northward mareh and have heen able to maintain themselyes on the outer islands where conditions closely approach those in more aretic regions.

The second wave was composed of the species of the Hudsonian and Canadian zones, the plants of the great coniferons forest areas. Although these plants may ocenr locally in southern New England as relic species, in the Penobscot Bay region they have an enviromment suited to their needs and here they are widespread.

The third wave to go northward consisted of anstral rather than boreal types. In all probibility this sonthern element formed a conspicuous part of the pre-glacial flora and like the northern forms returned to its old localities upon the withdrawal of the ice. This third invasion (if it may be so termed) of the glaciated region was probably augmented by other sonthern species which pressed northward from the original eenters of distribution for this flora in the southeast. This anstral flora consisted of two elements which followed separate channels in their northward migration: the Alleghanian plants going along the Appalachians and the Mississippi and Ohio Valleys, the Carolinian plants following the Atlantic Coastal Plain.

The first of these elements - the Alleghanian-reaches its best development on the hasic soils of the more temperate parts of the eastern Lnited States, a zone which in general harely reaches New England. We have already noted, how- 
ever, that certain Alleghanian speeies ocem locally in the Penobscot Bay area and also in considerable abundance in more northern regions. How was it possible for these plants of rich soils to eross the sterile areas of sonthern New England and reach the localities to the north where ther now ocenr! Fernald ${ }^{1}$ has called attention to the fact that the glacial till left behind at the receding of the ice was a mixed soil containing all the necessary elements of plant food. The Alleghanian plants wonld have been able to penetrate far to the north of their general range by utilizing the basie materials of this mixed soil. Conditions of elimate and the influence of the ocean also favored the adrance of these sontheru forms so that they were able to reach to Nova Scotia and Cape Breton.

The original continnous distribution of these anstral speeies on the drift area has been lost. As the basic elements became exhansted from the soil for one reason or another, this sonthern flora gradually beeame restricted to localities where the underlying rock furnished a soil with the proper requirements and where climatic conditions as well were particularly favorable. This gradual extinetion of Alleghanian speeies has been pronomneed in the eastern Penobseot Bay region. Originally the deeidnous trees were abundant but the forests were cut off by the early settlers. The consequent exposure of the soil to the sun and the processes of erosion, or the occurrence of fires, resulted in the destruction of the layer of humns necessary for the maintenance of the rich woods types. These species were perhaps able to persist for a while in the now unfavorable enviromment but soon became mable to compete snccessfully with the coniferous trpes which were everywhere encroaching on them, and were

1. Fernald. The Soil Preferences of Certain Alpine and Subalpine Plants. Rhodora 9: 149-193 (1907). 
soon restricted to the most favorable places. Small in his "History of Swans Island" describes this change in the type of forests on that island. "When the island was discovered by Europeans it was entirely eovered with a dense forest of hardwood trees which attained great size as shown by the enormons stumps found throughout the forest by the older inhabitants. After the first growth was cut off the land was burned over. This was injurious to the soil where it covered rocky land and much of its richness was washed into the valleys and thence into the oeean. The next growth on this impoverished soil was much smaller and can be remembered by many of the older inbabitants. When this forest was in turn eleared its place was taken by the stunted growth of spruce and fir which now covers the greater part of the island."

It is apparent that the Alleghanian plants, which in isolated localities have survived this extermination, represent relic species of a flora onee widespread. Is stated above, eertain Alleghanian species, other than the rich woods types, are more widely distributed in the region. These species, however, are more tolerant of soil conditions and are able to persist in some of the acid areas.

Probably contemporaneons with the preeding element the Carolinian element of the austral flora went northward along the coastal plain from centers of distribution in the sontheast. In its maximum development this coastal plain flora extends only to New Jersey. Britton ${ }^{2}$ and Hollick, however, have shown that certain of these coastal plain plants are found on

1. Small. A History of Swans Island, Maine. Ellsworth, 1898.

2. Britton. On the Northward Extension of the N. J. Pine Barren Flora on Long and Staten Islands. Bull Torr. Bot. Club $7: 81$ (1880).

3. Hollick. Annals N. Y. Acad. Sci. 11: 55 (1892); 13: 387 (1894). Trans. N. Y. Acad. Sci. 12: 189 (1893); 13:8 (1894). 
Long Island and in sontheastern New England; and more recently Fernald ${ }^{1}$ has demonstrated their necurrence in Newfoumdland. The arguments for this are too well known to need discussion here. Suffice it to say that there is good evidence of a land comnection directly after the Glacial Period, which reached from New Jersey to Cape Cod and thence to Tewfounlland and along which the enastal plain plants were able to migrate northward. This Carolinian element may have gone northward for the first time at the close of the Clacial Period, but in all prolability it existed in the Inth previous to this period and was driven sonth by the ice into New Jersey where it remained mil the retreat of the wacier and then retmrned orer the land-bridge. This landbridge has now been sulmerged or broken up and only isolated framments remain, each of which has a typical coastal plain flora.

Althongh this explanation holds good for the presence of Carolinian speeies in Tewfomdland and sontheastem New England it does not explain the ocenrence of such forms as Corema Conmatii and IIudsonia ericoides on the rncky granite smmmits of the Penobscot Pay islands. Between the land comnection atove referred to and the central portion of the Maine eoast there existed in early post-glacial times as at present, a deep and wide expanse of water, the (inlf of Maine. This must have been an effectual harrier to prevent any of the coastal plain speeies from reaching Maine by the land bridge. The only other ronte for these plants was across the glaciated areas of sonthern New England. We have alreary noted that the glacial till which covered this region was a mixed soil containing a great rariety of food elements. For plants which could exereise a

1. Fernald. A Botanical Expedition to Newfoundland and Southern Labrador. Rhodora 13: 109-163 (1911). 
selective absorption and take ont from the soil the necessary elements for their own mantenance, this till afforded a farorable habitat. The coastal plain plants, lowever, as mentioned carlier, are repelled by basic elements and are compelled to scek refuge on the most stcrite soils where these clements are lacking. Such plants would obvionsly have been muable to cross the glacial till, and their miform northward advance must have heen checked mutil the hasic materials had been removed from the soil. This might have been accomplished by the sinking of the coast, lnt we know from genlogical evidence that southern New England has not been under water since the Glacial Perind. The solution of the prollem is found by a study of the composite distribution of certain Carolinian species which have penctrated inland from the coastal region. These species extend up the river valleys and on the sand plains of eastern Massachnsetts to Tork County, Maine. An exannination of topographic maps of this general area shows that these sand plains are all old outwash plains.

Hills to the north dammed up the water from the melting ice at the close of the Glacial Period until the pressure liecanne too great. Then the barriers hurst and the water rushed over the surromding enuntry leeching out the soil as successfully as thongh it had heen more the ocean. The resulting out-wash plains now afforded a halitat suitalile for the adrance of the coastal plain species as far north as sonthcrn Maine. From there on the granite rocks of the enast furnished an equally silicious and sterile area on which the Carolinian plants pressed forward to the Penoleseot Bay region.

Not all anthorities agree as to the origin of the rarions

1. Woodworth. Some Glacial Wash-Plains of Southern New England. Essex Inst. Bull. 29: 71-119 (1897). 
elements of the flora of northeastern Ameriea. The greatest amount of evidenee, however, seems to be in favor of the theories outlined above, which are accepted by the majority of biologists, both botanists and zoologists. Among the disscnters from this view-point may he mentioned Scharff. In his "Origin of Life in America" he advances the theory that the pre-glacial flora was not driven south by the advance of the ice but survived the Glacial Perior in temperate islands in Labrador and Grecnland which escaped glaciation, from which areas it lias since spread to its present limits. The sonthern flora, on the other hand, according to Seharff, survived the Ice Age on the now submerged land bridge which extended from New Jersey to Cape Cod and Newfoundland and was likewise maffected by the glaciers.

There are differences of opinion on other points as well. In referring to the presence of arctie-alpine species on the summit of Mrt. Mrarey in the Adirondacks, Bray disclaims the theory that they are relic types, stating that it is not necessary to assume that the aretic-alpine flora of the high momtains was established at a remote period such as the close of the Glacial Period. "sinee factors at present operative might account for the earrying of such species to any habitat suited to them."

The geographic affinities of individual speeies also afford opportunities for differences of opinion. In general Corema Conradii and schizuea pusilla (to cite cxamples) are considered as southern coastal plain types which have pushed north. Stone, however, in his "Flora of Sonthern New .Jersey" ${ }^{\circ 3}$ elasses them as boreal speeies which have been driven sonth.

1. Scharff. Distributicn and Origin of Life in America. New York. 1912.

2. Bray. 1. c. pages 79-80.

3. Stone. The Plants of Southern New Jersey. Report of N. J. State Museum, 1911: 25-828. 


\section{THE INTRODL('ED) FLORA OF THE EASTERT PENOBS(')' B.AY REGION}

An account of the flora of the eastern Penobscot Bay region wonld not be complete withont some reference to the introdnced plants. These are much less abmudant than in most parts of the comntry and comprise only 13: species. This paneity is due ehiefly to the alsence of railroads or laree towns.

The most conspienons of the weeds of the area are plants which have a more or less northern trend, anong which may be mentioned Carum Corri and Matrienrin sunveolens along roadsides and abont houses: Hiemeinm prutense and $I I$. auruntiacum, too almundant in fields and pastures: Odontites mbre in dry fields; and senecio sylpaticus, eommon in gravelly soil at the edge of beaches and appearing almost indigenons.

The common weeds of the eistern Tnited states are also represented ly sneh species as:

Rumex Acetoselln

stellarin media

Capsella Bursa-pastoris Remunculus acris

Brassira nigra
Terbusenu Thapsus

C'Trysenthemum Leuconthemum.

var. pinnatifidum

Taraxacum officinate

sonrhus olerarus

Another type of introduced plants comprises fugitives from the West. The most interesting of these is Pluacelin linemis. a species of the Rocky Momntain region fomd in a balu-yard at Bronklin, where it had ofvionsly been introdnced in grain.

In addition to these plants, all of which ocemr spontanennsly, there are many garden escapes which have become well established, particularly in the neighborhool of old cellars or deserted honses. (often vignons specimens of Pyrus. Molus are seen in the midst of a forest which his re- 
dained a former orehard; while such species as syrmugn melyaris and Sorbaria sorbifolin frequently ocenr in old fields with such characteristic native plants as funiperus com-

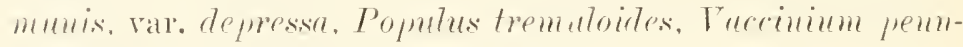
sylemirum and šmblucus racemose.

(WEIORY BOTANTEAL LABOIATOHY, YALE ITIVERITY. 




\title{
PORTLAND SOCIETY OF NATURAL HISTORY.
}

\author{
JOURNAL.
}

Vol. I, No. 1 (all ever issued). Morse. Pulmonifera of Maine. 63 pp., 10 pls., numerous text cuts. Few copies only.

$\$ 1.50$

\section{PROCEEDINGS.}

Vol. I, complete. 230 pp., 2 pls., 1 map, numerous cuts and index.

Vol. I. Part 1. $96+$ xiv pp., 1 pl., 1 map. Sold only with complete sets.

Vol. I, Part 2. $125+\mathrm{x}$ pp., 1 pl., numerous cuts and index to volume.

Cooke, M. C. Decades of Maine Fungi. 7 pp., numerous cuts (extract Proc., 1: ii). Few copies.

Billings, E. Fossils from Square Lake, Maine. $22 \mathrm{pp}$, folding plate (extract Proc., 1: ii). Few copies.

Wood, Wm. Clio borealis on the Coast of Maine. 3 pp., cut (extract Proc., 1: ii). Few copies.

Vol. II, complete. $282 \mathrm{pp}$., $5 \mathrm{pls}$, one cut, in text.

Vol. IT, in Parts.

Part 1. Brown, N. C. Rare Birds in the Vicinity of Portland. Only with complete sets.

Brown, N. C. Catalogue of Birds of Portland, with Supplement. 40 pp. Few copies only.

Part 2. Fernald, M. L. Catalogue of Maine Plants. 32 pp. $\$ .50$

Part 3. Fernald, II. L. Supplement to Catalogue of Naine Plants. $24 \mathrm{pp}$.

Part 4. Norton, A. H. Sharp-tailed Finches of Maine, and Norton, A. H. Ornithological Notes, $8 \mathrm{pp}$. $\$ .15$

Morton, F. S. Foraminifera of Marine Clays of Maine 18 pp., $1 \mathrm{pl}$.

Fernald, M. L. Second Supplement to Maine Plants. 15 pp. \$.15

Part 5. Norton, A. H. Birds Bowdoin Labrador Expedition. $20 \mathrm{pp}$. $1 \mathrm{pl}$.

Kingsley, J. S. Catalogue Marine Invertebrata of Casco Bay, Jlaine. $25 \mathrm{pp}$.

Manning, P. C. Glacial Pot-Holes in Maine. 15 pp., 2 pl. \$ .25

Part 6. Howe, R. H., ir. New Race of Microtus pennsylvanicus. 2 pp., 1 pl.

Part 7. Kingsley, J. S. Additions to Fecorded Fauna of Casco Bay. 3 pp.

Part \&. Kendall, W. C. Fishes of Labrador. 37 pp. \$. 40

Norton, A. H. Land and Fresh Water Crustaceans of Maine, 11 pp., 1 cut.

Part 9.- Collins, F. S. Marine Algae of Casco Bay. 25 pp. \$ .25

Vol. III, Part 1. Kendall, W. C. Fishes of Maine. \$1.50

Vol. III, Part 2. Hill, A F. Flora of Penboscot Bay Region, Pp. 10.5, euts and map. 


\section{MISCELLANEOUS PAPERS.}

Portland Catalogue of Maine Plants, 1868, 12 pp.

Wood, Wm. Vegetable Structure and the Importance of Certain Tissues in the Reparation of Wounds in Trees and Shrubs (May 2, 1881).

Hill, Thomas. Phyllotaxis.

Boyd, Chas. H. Walrus Remains at Addison, Maine. Stone, G. H. Glacial Erosion.

Lee, L. A. Account of the Work of the United States Fish Commission for the year 1881.

Stone, G. H. Columbite in Maine and Reports of Officers of the Society.

Report of the President, Dr. WWm. Wood, May 20, 1889, with Memoir on Rev. J. W. Chickering, former President. 


